\title{
Complementary Densities of Lévy Walks: Typical and Rare Fluctuations
}

\author{
A. Rebenshtok ${ }^{1}$, S. Denisov ${ }^{2,3,4}$, P. Hänggi ${ }^{2}$, E. Barkai ${ }^{1}$ * \\ ${ }^{1}$ Department of Physics, Institute of Nanotechnology and Advanced Materials \\ Bar-Ilan University, Ramat-Gan, 52900, Israel \\ ${ }^{2}$ Institute of Physics, University of Augsburg, Universitätsstrasse 1 \\ D-86135, Augsburg Germany \\ ${ }^{3}$ Sumy State University, Rimsky-Korsakov Street 2, 40007 Sumy, Ukraine \\ ${ }^{4}$ Department of Applied Mathematics, Lobachevsky State University of Nizhny Novgorod \\ Gagarin Avenue 23, 603950 Nizhny Novgorod, Russia
}

\begin{abstract}
Strong anomalous diffusion is a recurring phenomenon in many fields, ranging from the spreading of cold atoms in optical lattices to transport processes in living cells. For such processes the scaling of the moments follows $\left\langle|x(t)|^{q}\right\rangle \sim t^{q \nu(q)}$ and is characterized by a bilinear spectrum of the scaling exponents, $q \nu(q)$. Here we analyze Lévy walks, with power law distributed times of flight $\psi(\tau) \sim \tau^{-(1+\alpha)}$, demonstrating sharp bi-linear scaling. Previously we showed that for $\alpha>1$ the asymptotic behavior is characterized by two complementary densities corresponding to the bi-scaling of the moments of $x(t)$. The first density is the expected generalized central limit theorem which is responsible for the low-order moments $0<q<\alpha$. The second one, a non-normalizable density (also called infinite density) is formed by rare fluctuations and determines the time evolution of higher-order moments. Here we use the Faà di Bruno formalism to derive the moments of sub-ballistic super-diffusive Lévy walks and then apply the Mellin transform technique to derive exact expressions for their infinite densities. We find a uniform approximation for the density of particles using Lévy distribution for typical fluctuations and the infinite density for the rare ones. For ballistic Lévy walks $0<\alpha<1$ we obtain mono-scaling behavior which is quantified.
\end{abstract}

Keywords and phrases: superdiffusion, Lévy walks, large deviations, infinite densities, strong anomalous diffusion, bi-fractal

Mathematics Subject Classification: 35Q53, 34B20, 35G31

\section{Introduction}

Many theories have been devised and experiments done on particle diffusion in a medium, when all particles start from the same origin. Typically, the corresponding dispersal process is characterized by the time evolution of the mean square displacement (MSD) $\left\langle x^{2}\right\rangle \sim t^{\xi}$, where for $\xi>1$ it is termed super-diffusion, and for $\xi<1$, sub-diffusion. It is well known by now that the MSD gives only partial

\footnotetext{
${ }^{*}$ Corresponding author. E-mail: barkaie@biu.ac.il
} 
characterization of the underlying stochastic process, though of-course Gaussian processes with no-drift, encountered frequently in the laboratory, e.g., Brownian motion, are an exception. For such processes a knowledge of the MSD fully determines the spatial density of particles $P(x, t)$, which also represents a normalized probability density in the framework of probability theory.

A more sophisticated way to analyze a diffusive processes is to study the time evolution of a continuous set of its moments, $\left\langle|x|^{q}(t)\right\rangle, q \geq 0$. The moments grow in time, and this growth can be characterized by a spectrum of exponents,

$$
\left\langle|x|^{q}(t)\right\rangle \sim M_{q} t^{q \nu(q)},
$$

where $q \geq 0$. For the normal Brownian motion $\nu(q)=1 / 2$. When $\nu(q)$ is not a constant, the process is said to exhibit strong anomalous diffusion [15,41,42]. A nonlinear spectrum of exponents, $q \nu(q)$, is an indication that the asymptotic density of particles is not determined by a unique scaling exponent.

$$
P(x, t) \neq \frac{1}{t^{\nu}} g\left(\frac{x}{t^{\nu}}\right)
$$

in the sense that mono-scaling predicts a constant $\nu(q)$. Thus if one finds (for example in an experiment) a nonlinear spectrum, then certain popular fractional diffusion equations [37], the fractional Brownian motion [35], and the central limit theorem, which are all mono-scaling theories, are either invalid or insufficient to handle the long-time moment evolution. It therefore becomes clear that one should go beyond the central limit theorem in order to understand the origin of the bi-scaling phenomena.

A particular type of strong anomalous diffusion, which has been detected in many natural dispersive phenomena, is characterized by the piecewise linear exponent spectrum [15]

$$
q \nu(q)=\left\{\begin{array}{rl}
d_{1} q & q<q_{c} \\
d_{2} q+d_{3} & q>q_{c}
\end{array}\right.
$$

The function $q \nu(q)$ is continuous and increasing with $q$, and there is a breaking point $q_{c}$, the solution of the linear equation $d_{1} q_{c}=d_{2} q_{c}+d_{3}, d_{1}, d_{2}>0$. For example, $d_{1}=1 / 2, d_{2}=1, d_{3}=-1$ and $q_{c}=2$. In this example the low moments $q<q_{c}$ exhibit normal scaling, similar to Brownian motion, while the fact that $d_{2}=1$ indicates that high order moments exhibit ballistic scaling $x \sim t . d_{3}$ acts to maintain the continuity of the spectrum, though the derivative of $q \nu(q)$ is not continuous. For a particular Lévy walk model the spectrum $q \nu(q)$ is shown in Fig. 1.

Phenomena with a spectrum Eq. (1.3) are sometimes called bi-scaling or bi-fractal processes. They are widely observed in a broad range of systems, for example Lagrangian motion in time-dependent incompressible velocity fields [15], intermittent maps and other nonlinear systems [1,30], Lorentz gas with an infinite horizon [10,44], sand pile models [14], systems with quenched disorder used to mimic diffusion of light in a Lévy glass $[3,11,12]$ and statistics of occupation times in renewal theory [25]. Piecewise bi-scaling behavior of $q \nu(q)$ was found in experiments of particles diffusing in a live cell, the strong anomalous diffusion being related to the active (i.e. non-thermal and non-Brownian) motion in the cell [24]. Strong anomalous diffusion was very recently found in super-diffusion of membrane targeting $\mathrm{C} 2 \mathrm{~A}$ domains on a lipid bilayer, and the relation to the Lévy walk model demonstrated experimentally [31] (see also [13]). Lately, similar phenomena were also observed in models of diffusion of cold atoms in optical lattices [28], in flows in porous media [18] and in the fluctuations around equilibrium laws in continuous time random walks [45].

The bi-scaling of the moments implies (roughly speaking) bi-scaling of the packet of particles [41,42]. This dual nature means, as we will show, a density profile describing diffusive bulk fluctuations, while a quasi-ballistic scaling describes the tails of the cloud of spreading particles. In this paper we analyze this behavior using a stochastic framework. Using the Lévy walk model [54] we show that the well known piecewise bi-scaling is related to two spatial densities. The low order moments $q<q_{c}$ originate from the Gauss-Lévy central limit theorem. The higher order moments $q>q_{c}$ are related to an infinite density (ID), meaning, it is an unnormalized density. None the less, we show that this mathematical 


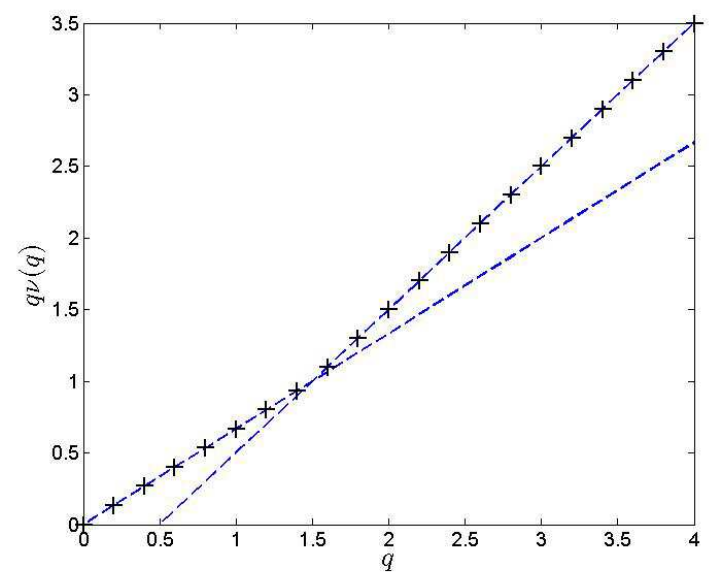

FIgURE 1. Bilinear spectrum of scaling exponents $q \nu(q)$ versus $q$ for a sub-ballistic superdiffusive Lévy walk. Crosses present numerical simulation results for the two state model with the waiting-time PDF given by Eq. (2.2), $\alpha=3 / 2$ and for time $t=10^{5}$. Dashed lines correspond to linear dependencies $q \nu(q)=q / \alpha$ and $q \nu(q)=q+1-\alpha$. The two lines intersect at the point $q_{c}=\alpha$, see equation (1.3).

object describes a large class of observables, i.e., observables that are integrable with respect to that non-normalized density. Further more, the infinite density describes the packet of particles when scaled properly, and hence it describes physical reality. The infinite density is thus a complementary tool to the central limit theorem. We finally give a uniform approximation, based both on the central limit theorem and the infinite density. The former describes the short scales of the problem and the latter the large scales. Since $q_{c}<2$ (for super-diffusion see below) even the second moment, traditionally considered as the defining moment of symmetric fluctuations, is an observable integrable with respect to the infinite density, so not surprisingly for a system with power law statistics, characterization of the tail must be made precise.

The Lévy walk model has gained recent new interest in the dynamics of cold atoms in optical lattices $[6,28]$, and the spreading of heat in nonlinear lattices $[20,52,53]$. New work emerged on its stochastic formalism in particular on the non-linear coupling between jump size and waiting times $[2,19]$. Lévy walks have a wide range of applications, at-least for a first approximation of a stochastic phenomenon. This includes blinking quantum dots [36,47], diffusion of tracer particles in turbulent flows [46], among other examples (see review [54]). Here we use stochastic tools based on the renewal assumption without attempting to justify the concept of infinite densities from a microscopical point of view. Considerable mathematical work was devoted for the classification of Lévy walks and related processes, for example the limit theorems and governing equations were investigated in $[5,8,21,27,34,48]$. However as far as we know the concept of infinite densities was not promoted in the Lévy walk context within the mathematical literature.

The density of particles, $P(x, t)$, is normalized to unity for any fixed time, i.e., $\int_{-\infty}^{\infty} P(x, t) \mathrm{d} x=1$. This is merely the reflection of conservation of matter, i.e., particles are not created neither annihilated in our system. Since normalization is conserved, one tends to believe that a non-normalized density cannot turn to a useful concept [43]. Maybe that is the reason why the statistics of the Lévy walk model were not completely worked out so far (though much is known of-course). One of our aims is to remove the mystery from the concept of infinite densities, at least within the context of the Lévy walk model. Infinite invariant densities are used in infinite ergodic theory, though their application into statistical physics is only recent, see, for example [28]. 
Non-normalized densities for Lévy walks were introduced in our recent publications [41, 42]. Here we provide a new method of calculating the infinite density using the Mellin transform technique, and provide a detailed derivation of the moments of the process, needed for the full characterisation of the anomalous statistics.

Bilinear scaling is not observed in the ballistic phase of the motion, namely, when the average flight time diverges. For that case $q \nu(q)=q$ and then a mono-scaling theory is valid. Here we provide an expression for the density of of particles based on an integral representation. While this was the subject of a recent research presented in Ref. [23], here we address some additional cases not considered so far; for example, the cases of non-symmetric and biased ballistic walks.

\section{The models}

In this section we describe the Lévy walk models while observing the position of a particle, $x(t)$, at time $t$. Two models will be presented: the velocity and the jump models. These two models are treated using the tools of renewal theory [25]. In order to distinguish between the models, we use $\cdots$ notation to mark observables and functions for the jump model. Therefore, for example, $x(t)$ refers to the velocity model while $\tilde{x}(t)$ relates to the jump model.

\subsection{The velocity model}

A particle, traveling with constant velocity, undergoes renewal events which alter its velocity. These events, e.g., collisions, take place in the time interval $(0, t)$. Between collisions, the particle travels ballistically with velocity $v_{j-1}$ for the duration of the sojourn time, $\tau_{j}$. The $\left\{v_{j}\right\}_{j=0,1, \ldots}$ and $\left\{\tau_{j}\right\}_{j=1,2, \ldots}$ are identically and independently distributed (iid) random variables with probability density functions (PDF) $F(v)$ and $\psi(\tau)$ respectively. We assume that $F(v)$ is symmetric around $v=0$, so all odd moments are zero and all of its even moments finite. For example, the two state model

$$
F_{\mathrm{TS}}(v)=\left[\delta\left(v-v_{\mathrm{c}}\right)+\delta\left(v+v_{\mathrm{c}}\right)\right] / 2,
$$

where $v_{\mathrm{c}}>0$. Most importantly, the PDF of the waiting time is given in the long time limit by

$$
\psi(\tau) \sim \frac{A}{|\Gamma(-\alpha)|} \tau^{-1-\alpha},
$$

with $A>0$ and $\alpha>0$. For the case $0<\alpha<1$ the mean waiting time diverges, where as for $1<\alpha<2$ there exists a finite mean, $\langle\tau\rangle=\int_{0}^{\infty} \tau \psi(\tau) \mathrm{d} \tau$, yet the second moment diverges. We will treat the case of $0<\alpha<1$ in Sec. 8 while now we focus on the latter. For applications in the next subsections, note the expansion of the one sided Laplace transform in the small $u$ limit for the $1<\alpha<2$ case $[7,25,29,37,55]$ which is

$$
\hat{\psi}(u)=\int_{0}^{\infty} e^{-u \tau} \psi(\tau) \mathrm{d} \tau \sim 1-\langle\tau\rangle u+A u^{\alpha} \ldots
$$

We define $x(t)$, the position of the particle at time $t$ as

$$
x(t)=\sum_{j=1}^{n} v_{j-1} \tau_{j}+v_{n} \tau_{b},
$$

where $n$ is a random non-negative integer referring to the number of renewal events during $(0, t)$ (see Fig. 2). Each event can be explained as a collision, such that the $n$th collision event occurs at time $t_{n}=\sum_{j=1}^{n} \tau_{j}$ and $\tau_{b}=t-t_{n}$ is the backward recurrence time [25].

The PDF, $P(x, t)$, of the particle's position $x$ at time $t$ describes the system. We present simpler and known expressions in the $(k, u)$ space, with $(k, x)$ as the Fourier conjugate pair and $(u, t)$ as the Laplace conjugate pair. The Fourier-Laplace transform is defined as

$$
P(k, u)=\int_{0}^{\infty} \mathrm{d} t \int_{-\infty}^{\infty} \mathrm{d} x e^{i k x-u t} P(x, t) .
$$


In what follows we use the convention of indicating the space we are working in by the function's variables in the parenthesis.

The Montroll-Weiss equation [7,29,55], describing the process in the $(k, u)$ space, is given by

$$
P(k, u)=\left\langle\frac{1-\hat{\psi}(u-i k v)}{u-i k v}\right\rangle \frac{1}{1-\langle\hat{\psi}(u-i k v)\rangle} .
$$

Here $\langle\cdot\rangle$ indicates averaging with respect to $v$ only. The full derivation is given in App. A.

\subsection{The jump model}

In the jump model the particle jumps only at the epoch of the next event, holding still during the sojourn time (see Fig. 2). Mostly static, the $j$ th jump is proportional to the sojourn time $\tau_{j}$ multiplied by a factor $v_{j-1}$ with velocity units. The position, $\tilde{x}(t)$, differs from Eq. (2.4) by the omission of the backward recurrence movement,

$$
\tilde{x}(t)=\sum_{j=1}^{n} v_{j-1} \tau_{j} .
$$

With no movement in the backward recurrence time, one of our goals is to examine its effect on the $P(x, t)$, i.e., the differences between the two models. Clearly in normal processes this is negligible in the long time limit. For a power law $\psi(\tau)$ the effects of movement during the backward recurrence time are macroscopically noticeable $[7,23]$.

The Fourier-Laplace transform of the PDF, $\tilde{P}(x, t)$, is derived in App. B, termed the Montroll-Weiss equation for the jump model,

$$
\tilde{P}(k, u)=\frac{1-\hat{\psi}(u)}{u} \frac{1}{1-\langle\hat{\psi}(u-i k v)\rangle} .
$$

\section{Integer moments for $1<\alpha<2$}

In this section, we investigate the long time limit of the moments of the process for $1<\alpha<2$ using the Montroll-Weiss equation. These moments will later be used to derive the infinite density.

\subsection{The moments of the velocity model}

The long time limit is investigated with the standard [37] small Laplace variable $u$ behavior of Eq. (2.3) inserted into Eq. (2.6)

$$
P(k, u) \sim \frac{1-A_{\tau}\left\langle[u-i k v]^{\alpha-1}\right\rangle}{u-A_{\tau}\left\langle[u-i k v]^{\alpha}\right\rangle},
$$

where $A_{\tau}=A /\langle\tau\rangle$.

The moments $\left\langle x^{m}(u)\right\rangle$ are given by the general Leibniz rule of derivation:

$$
\begin{aligned}
& \left\langle x^{m}(u)\right\rangle=\left.\left.\frac{\partial^{m} P(k, u)}{\partial(i k)^{m}}\right|_{k=0} \sim \sum_{j=0}^{m}\left(\begin{array}{c}
m \\
j
\end{array}\right) \frac{\partial^{m-j}}{\partial(i k)^{m-j}}\left(1-A_{\tau}\left\langle[u-i k v]^{\alpha-1}\right\rangle\right) \frac{\partial^{j}}{\partial(i k)^{j}} \frac{1}{u-A_{\tau}\left\langle[u-i k v]^{\alpha}\right\rangle}\right|_{k=0} \\
= & \left.\frac{\partial^{m}}{\partial(i k)^{m}} \frac{1}{u-A_{\tau}\left\langle[u-i k v]^{\alpha}\right\rangle}\right|_{k=0}-\left.A_{\tau} \sum_{j=0}^{m}\left(\begin{array}{c}
m \\
j
\end{array}\right) \frac{\partial^{m-j}}{\partial(i k)^{m-j}}\left\langle[u-i k v]^{\alpha-1}\right\rangle \frac{\partial^{j}}{\partial(i k)^{j}} \frac{1}{u-A_{\tau}\left\langle[u-i k v]^{\alpha}\right\rangle}\right|_{k=0} .
\end{aligned}
$$




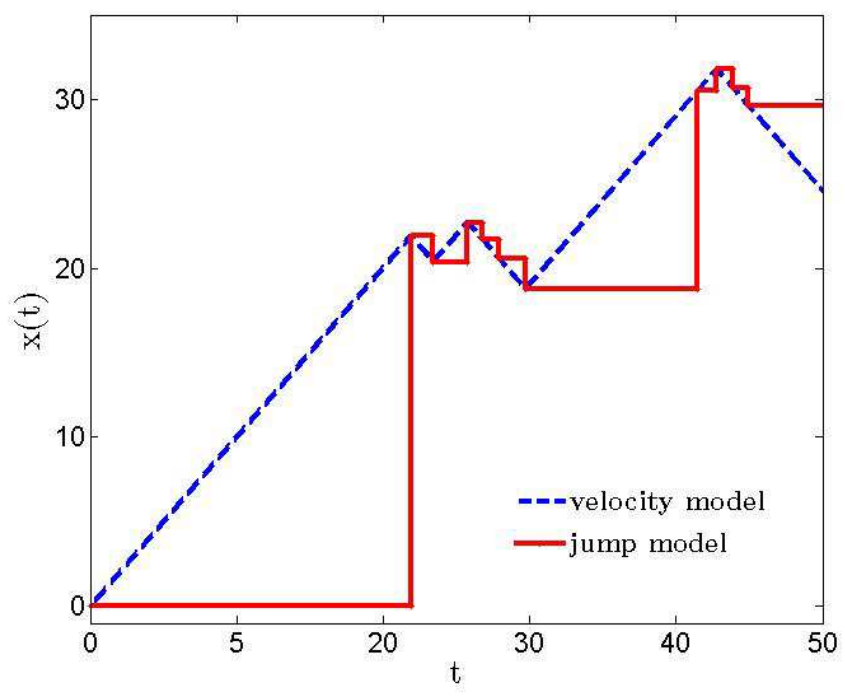

Figure 2. (color online) A sketch of trajectories for two Lévy walk models. For the two state velocity model the particle's velocity attains values \pm 1 with equal probabilities. Hence for the velocity model (dotted line), the value of $x(t)$ either increases or decreases continuously in time, in the course of ballistic motion events. In the jump model (solid line), $\widetilde{x}(t)$ is discontinuous and it is updated at the end of each waiting event. The sequence of the collision times $t_{1}, t_{2}, \ldots$ is the same for both models.

The exact expression for the derivative of the denominator is given by the Faà di Bruno formula [33]

$$
\left.\frac{\partial^{j}}{\partial(i k)^{j}} \frac{1}{u-A_{\tau}\left\langle[u-i k v]^{\alpha}\right\rangle}\right|_{k=0}=\sum_{z=1}^{j} \frac{(-1)^{z} z !}{\left(u-A_{\tau} u^{\alpha}\right)^{z+1}} B_{j, z}\left(\theta_{1}, \theta_{2}, \ldots, \theta_{j-z+1}\right),
$$

where $B_{j, z}\left(\theta_{1}, \theta_{2}, \ldots \theta_{j-z+1}\right)$ is the partial Bell polynomial [17]

$$
B_{j, z}\left(\theta_{1}, \theta_{2}, \ldots, \theta_{j-z+1}\right)=\sum \frac{j !}{r_{1} ! r_{2} ! \ldots r_{j-z+1} !}\left(\frac{\theta_{1}}{1 !}\right)^{r_{1}}\left(\frac{\theta_{2}}{2 !}\right)^{r_{2}} \cdots\left(\frac{\theta_{j-z+1}}{(j-z+1) !}\right)^{r_{j-z+1}}
$$

and the summation is taken over all the sequences of integers $r_{1}, r_{2}, \ldots \geq 0$ such that:

$$
\left\{\begin{array}{r}
r_{1}+r_{2}+\ldots r_{j-z+1}=z \\
r_{1}+2 r_{2}+\ldots(j-z+1) r_{j-z+1}=j
\end{array}\right.
$$

The $\left\{\theta_{z}\right\}_{z=1,2 \ldots}$ in Eq. (3.3) are given by

$$
\theta_{z}=\left\{\begin{aligned}
-A_{\tau}(-\alpha)_{z}\left\langle v^{z}\right\rangle u^{\alpha-z} & \text { even } z \\
0 & \text { odd } z
\end{aligned}\right.
$$

with $(\alpha)_{z}=\alpha(\alpha+1) \cdots(\alpha+z-1)$, the Pochhammer polynomial. Non-zero valued partial Bell polynomials are obtained by setting $r_{1}=r_{3}=\ldots=0$, meaning $j$ must be even.

Using a partial Bell polynomials identity derived from Eqs. (3.4) and (3.5) we find

$$
B_{j, z}\left(\theta^{a+b}, \theta^{a+2 b}, \ldots, \theta^{a+(j-z+1) b}\right)=\theta^{z a+j b} B_{j, z}(1,1 \ldots) .
$$


We simplify the partial Bell polynomial in Eq (3.3) using $\vartheta_{z} \equiv(-\alpha)_{z}\left\langle v^{z}\right\rangle$,

$$
B_{j, z}\left(\theta_{1}, \theta_{2}, \ldots\right)=(-1)^{z} A_{\tau}^{z} u^{z \alpha-j} B_{j, z}\left(0, \vartheta_{2}, 0, \vartheta_{4}, \ldots\right) .
$$

We now find the long time behavior of the moments, Eq. (3.2), which corresponds to the small $u$ limit

$$
\left\langle x^{m}(u)\right\rangle \sim \sum_{j=1}^{m} \frac{A_{\tau}^{j} j ! B_{m, j}\left(0, \vartheta_{2}, \ldots\right)}{u^{1+m-j(\alpha-1)}}-\frac{\left\langle v^{m}\right\rangle(1-\alpha)_{m} A_{\tau}}{u^{2+m-\alpha}}-\sum_{j=1}^{m}\left(\begin{array}{c}
m \\
j
\end{array}\right) \sum_{z=1}^{j} \frac{\left\langle v^{m-j}\right\rangle(1-\alpha)_{m-j} A_{\tau}^{z+1} z ! B_{j, z}\left(0, \vartheta_{2}, \ldots\right)}{u^{2+m-\alpha-z(\alpha-1)}},
$$

It is clear from symmetry that for odd $m$ th moments $\left\langle x^{m}(t)\right\rangle=0$. For even $m$, the leading term of Eq. (3.9), in the small $u$ limit, is $u^{-m-2+\alpha}$. We perform the inverse Laplace transform, $u^{\alpha-m-2} \leftrightarrow$ $t^{m+1-\alpha} / \Gamma(m+2-\alpha)$, and find in the long time limit

$$
\left\langle x^{m}(t)\right\rangle \sim \mathcal{B} \frac{m\left\langle v^{m}\right\rangle}{(m-\alpha)(m-\alpha+1)} t^{m+1-\alpha},
$$

with $m=2,4,6,8, \ldots$ and

$$
\mathcal{B}=A /[\langle\tau\rangle|\Gamma(1-\alpha)|]
$$

Eq. (3.10) relates the moments of $\left\langle x^{m}(t)\right\rangle$ with the velocity moments of $F(v)$ and with $A /\langle\tau\rangle$ and $\alpha$, the asymptotic parameters of $\psi(\tau)$, Eq. (2.3). For $m=2$ we find super-diffusion $\left\langle x^{2}(t)\right\rangle \sim t^{3-\alpha}$ which is well known. So far we did not detect strong anomalous diffusion, this is merely due the fact that as we show below the critical moment $q_{c}=\alpha<2$, while here we have considered integer moments $m=2, \ldots$.

\subsection{The moments of the jump model}

For the jump model we insert the expansion $\hat{\psi}(u)$, Eq. (2.3), to the Montroll-Weiss equation, Eq. (2.8), yielding

$$
\tilde{P}(k, u) \sim \frac{1-A_{\tau} u^{\alpha-1}}{u-A_{\tau}\left\langle[u-i k v]^{\alpha}\right\rangle} .
$$

We now turn to calculate the asymptotic moments $\left\langle\tilde{x}^{m}(u)\right\rangle$. The nominator's independence of $k$ in Eq. (3.12) simplifies the calculations. Using Eqs. (3.2) and (3.3) we find

$$
\left\langle\tilde{x}^{m}(u)\right\rangle=\left.\left.\frac{\partial^{m} \tilde{P}(k, u)}{\partial(i k)^{m}}\right|_{k=0} \sim\left(1-A_{\tau} u^{\alpha-1}\right) \frac{\partial^{m}}{\partial(i k)^{m}} \frac{1}{u-A_{\tau}\left\langle[u-i k v]^{\alpha}\right\rangle}\right|_{k=0}=\left(1-A_{\tau} u^{\alpha-1}\right) \sum_{j=1}^{m} \frac{(-1)^{j} j ! B_{m, j}\left(0, \theta_{2}, \ldots\right)}{\left(u-A_{\tau} u^{\alpha}\right)^{j+1}} .
$$

Using Eq. (3.8), the expression is further simplified in the small $u$ limit yielding

$$
\left\langle\tilde{x}^{m}(u)\right\rangle \sim \sum_{j=1}^{m} \frac{A_{\tau}^{j} j ! B_{m, j}\left(0, \vartheta_{2}, \ldots\right)}{u^{1+m-j(\alpha-1)}}=\frac{A_{\tau} B_{m, 1}\left(0, \vartheta_{2}, \ldots, \vartheta_{m}\right)}{u^{2+m-\alpha}}+O\left(\frac{1}{u^{3+m-2 \alpha}}\right),
$$

where $B_{m, 1}\left(\vartheta_{1}, \ldots, \vartheta_{m}\right)=\vartheta_{m}$ with $r_{m}=1$ and its predecessors are zero according to Eqs. (3.4) and (3.5). Applying the inverse Laplace transform, we find for even values of $m$,

$$
\left\langle\tilde{x}^{m}(t)\right\rangle \sim \mathcal{B} \frac{\alpha\left\langle v^{m}\right\rangle}{(m-\alpha)(m-\alpha+1)} t^{m+1-\alpha} .
$$

Notice that for every $m$ the moments of the velocity and jump models uphold $\left\langle x^{m}(t)\right\rangle \geq\left\langle\tilde{x}^{m}(t)\right\rangle$. This occurs since the velocity model includes the distance traveled during the backward recurrence time $\tau_{b}$, where as in the jump model the particle is grounded up until $t_{n+1}$, the end of the $n$th event (see Fig. 2). 


\section{The infinite density}

In the last section we obtained two asymptotic expressions for the even moments. Using heuristics and the inverse Mellin transform we plan to derive a matching density and examine its traits. As we shall see, the density so derived is an infinite density (ID).

\subsection{The inverse Mellin transform approach}

The Mellin transform is related to the Laplace and Fourier transforms [38]. It maps a function $B(v)$ on the positive real axis $v>0$, to a function $M(q)$ defined on the complex plane, $q$,

$$
M(q)=\int_{0}^{\infty} v^{q-1} B(v) d v
$$

$M(q)$ is the Mellin transform of $B(v)$ and is defined on the strip of definition, $\mathbb{S}$, for every $q \in \mathbb{S}$, for which convergence of $M(q)$ is guarantied [16,38]. Clearly, if $B(v)$ is a PDF with all its moments finite then the strip of definition will include $1 \leq R e(q)$ and $M(q)=\left\langle v^{q-1}\right\rangle$. The inverse Mellin transform [38] is given by

$$
B(v)=\frac{1}{2 \pi i} \int_{c-i \infty}^{c+i \infty} M(q) \frac{d q}{v^{q}},
$$

where $c \in \mathbb{S}$. These definitions are easily extended to the case of a symmetric PDF over $v \neq 0$ with $M(q+1)=\left\langle|v|^{q}\right\rangle / 2$. In our case, since $F(v)$ is symmetric so we can simply use Eq. (4.2) to write it in terms of the inverse Mellin transform $\left\langle|v|^{q}\right\rangle / 2$,

$$
F(v)=\frac{1}{4 \pi i} \int_{c-i \infty}^{c+i \infty} \frac{\left\langle|v|^{q}\right\rangle}{|v|^{q+1}} \mathrm{~d} q,
$$

where $\operatorname{Re}(q)=c \geq 0$ is included in the strip of definition [38], i.e., the domain for which $\left\langle|v|^{q}\right\rangle$ is analytic. In practice, we obtain $\left\langle|v|^{q}\right\rangle$ for $\operatorname{Re}(q) \geq 0$ and now continue this function in the whole complex plane. This procedure gives $\left\{q_{j}\right\}_{j=1 \ldots n_{q}}$ as the poles of $\left\langle|v|^{q}\right\rangle$ which are located at $\operatorname{Re}(q)<0$ (see details below). A complementary example is the case where $F(v)$ is composed of only Dirac delta functions such that for the two state velocity model, where $v= \pm v_{\mathrm{c}}$ with equal probability, $\left\langle|v|^{q}\right\rangle=\left|v_{\mathrm{c}}\right|^{q}$ has no poles.

We intend to use the inverse Mellin transform to extract from the moments $\left\langle|x(t)|^{q}\right\rangle$ a density, $\mathcal{I}_{\mathrm{d}}(x / t)$, which will turn out to be the ID. The inverse Mellin transform is suited to extract a generic form of the infinite density since it operates directly on the moments, unlike the inverse Fourier based method [42], which includes two steps: a Taylor series summation of the integer moments and an inverse Fourier integral applied to it. In order to continue we acknowledge that Eq. (3.10) can be analytically continued as absolute value moments, i.e., Eq. (3.10), can be used to calculate the long time dependence of $\left\langle|x(t)|^{q}\right\rangle$ for $q>\alpha$,

$$
\left\langle|x(t)|^{q}\right\rangle \sim \mathcal{B} \frac{q\left\langle|v|^{q}\right\rangle}{(q-\alpha)(q-\alpha+1)} t^{q+1-\alpha} .
$$

The analytic continuation assumption is validated later by theoretical arguments (e.g. the matching the density tail and bulk solutions, see below) and simulations. The choice of $q>\alpha$ is inferred from the strip of definition of $\left\langle|v|^{q}\right\rangle$, Eq. (4.3) and the two poles: $q=\alpha-1$ and $q=\alpha$, since we must have valid moments from some critical order value and on.

We first define

$$
\frac{\left\langle|x(t)|^{q}\right\rangle}{2 t^{q+1-\alpha}} \sim \int_{0}^{\infty} \bar{v}^{q} \mathcal{I}_{\mathrm{d}}(\bar{v}) d \bar{v},
$$

where

$$
\bar{v}=\frac{x}{t}=\frac{1}{t} \int_{0}^{t} v(t) \mathrm{d} t
$$


the time-averaged velocity. Note that in Eq. (4.5) we have exploited the symmetry of the problem, writing the integral from 0 to $\infty$, the factor $1 / 2$ on its left hand side stems from that same symmetry. The inverse Mellin transform is given by

$$
\mathcal{I}_{\mathrm{d}}(\bar{v}) \sim \frac{1}{4 \pi i} \int_{c-i \infty}^{c+i \infty} \frac{\left\langle|x(t)|^{q}\right\rangle}{t^{q+1-\alpha}} \frac{d q}{|\bar{v}|^{q+1}} .
$$

Inserting $\left\langle|x(t)|^{q}\right\rangle$, Eq. (4.4), results in

$$
\mathcal{I}_{\mathrm{d}}(\bar{v})=\frac{\mathcal{B}}{4 \pi i} \int_{c-i \infty}^{c+i \infty} \frac{q}{(q-\alpha)(q-\alpha+1)} \frac{\left\langle|v|^{q}\right\rangle}{|\bar{v}|^{q+1}} \mathrm{~d} q
$$

In both cases the strip of definition is $c>\alpha$ due to the verified analytic property of $\left\langle|v|^{q}\right\rangle$, Eq. (4.3), in that region and the poles at $q=\alpha-1$ and $\alpha$.

We demonstrate the use of Eq. (4.8) to calculate $\mathcal{I}_{\mathrm{d}}(\bar{v})$. We solve the inverse Mellin transform integral using the calculus of residues [4] for a specific case, the two state velocity model, Eq. (2.1). The ID, $\mathcal{I}_{\mathrm{TS}}(\bar{v})$, is

$$
\mathcal{I}_{\mathrm{TS}}(\bar{v})=\frac{\mathcal{B}}{4 \pi i} \int_{c-i \infty}^{c+i \infty} \frac{q}{(q-\alpha)(q-\alpha+1)} \frac{\left|v_{\mathrm{c}}\right|^{q}}{|\bar{v}|^{q+1}} \mathrm{~d} q,
$$

with $c>\alpha$. For $|\bar{v}| \leq v_{\mathrm{c}}$ one notices that in order to solve the integral using the residues theorem, we should use the closed contour plotted in Fig. 3A, since the other sections: the horizontal sections, $\operatorname{Im}(q) \rightarrow \pm \infty$, and $\operatorname{Re}(q) \rightarrow-\infty$ do not contribute,

$$
\mathcal{I}_{\mathrm{TS}}(\bar{v})=\left.\frac{\mathcal{B}}{2}\left[\frac{q}{(q-\alpha+1)} \frac{\left|v_{\mathrm{c}}\right|^{q}}{|\bar{v}|^{q+1}}\right]\right|_{q=\alpha}+\left.\frac{\mathcal{B}}{2}\left[\frac{q}{(q-\alpha)} \frac{\left|v_{\mathrm{c}}\right|^{q}}{|\bar{v}|^{q+1}}\right]\right|_{q=\alpha-1}=\frac{\alpha \mathcal{B}}{2 v_{\mathrm{c}}}\left|\frac{v_{\mathrm{c}}}{\bar{v}}\right|^{\alpha+1}\left(1-\frac{\alpha-1}{\alpha}\left|\frac{\bar{v}}{v_{\mathrm{c}}}\right|\right) .
$$

For $|\bar{v}|>v_{\mathrm{c}}$, the vertical branch at $\operatorname{Re}(q) \rightarrow-\infty$ gives a nonzero contribution and thus we change contours to the one plotted in Fig. 3B. Without any residues inside the contour the result is $\mathcal{I}_{\mathrm{TS}}(\bar{v})=0$. The two state model is the simplest case one can analyze. A general symmetric velocity PDF, $F(v)$, which yields poles on the left hand side of the plane, $\operatorname{Re}(q)<0$, allows retaining the same contour presented in Fig. $3 \mathrm{~A}$ for all values of $\bar{v} \neq 0$. Also, notice for further use the archetype function

$$
\mathcal{I}_{\mathcal{T S}}(\bar{\nu})=v_{c} \mathcal{I}_{\mathrm{TS}}\left(v_{\mathrm{c}} \bar{\nu}\right)
$$

where $\bar{\nu}$ is unit-less.

Using Eq. (4.8) and assuming that $q=\alpha-1$ and $q=\alpha$ are 1st order poles and $F(v)$ is not composed of Dirac delta functions, we calculate the general case infinite density over the extended closed contour plotted in Fig. 3A,

$$
\begin{aligned}
\mathcal{I}_{\mathrm{d}}(\bar{v}) & =\frac{\alpha \mathcal{B}}{2} \frac{\left\langle|v|^{\alpha}\right\rangle}{|\bar{v}|^{\alpha+1}}\left(1-\frac{(\alpha-1)}{\alpha} \frac{\left\langle|v|^{\alpha-1}\right\rangle}{\left\langle|v|^{\alpha}\right\rangle}|\bar{v}|\right) \\
& +\frac{\mathcal{B}}{2} \sum_{j=1}^{n_{q}}\left[\frac{q_{j} \operatorname{Res}\left(\left\langle|v|^{q}\right\rangle, q_{j}\right)}{\left(q_{j}-\alpha\right)\left(q_{j}-\alpha+1\right)}|\bar{v}|^{-q_{j}-1}\right],
\end{aligned}
$$

where $\operatorname{Res}\left(\left\langle|v|^{q}\right\rangle, q_{j}\right)$ is given by

$$
\operatorname{Res}\left(\left\langle|v|^{q}\right\rangle, q_{j}\right)=\frac{1}{2 \pi i} \oint_{R \rightarrow 0}\left\langle|v|^{q_{j}+z}\right\rangle \mathrm{d} z
$$

and $R$, the radius of the circle which encircles the pole $q_{j}$. We identify in the general expression, Eq. (4.12) three parts: (i) the $|\bar{v}|^{-1-\alpha}$ term, (ii) the $|\bar{v}|^{-\alpha}$ term and (iii) a polynomial of $|\bar{v}|$. The first two, which stem from the poles $\alpha-1$ and $\alpha$. In Sec. 4.3 we give an asymptotic integral expression replacing the polynomial for large $|\bar{v}|$. 

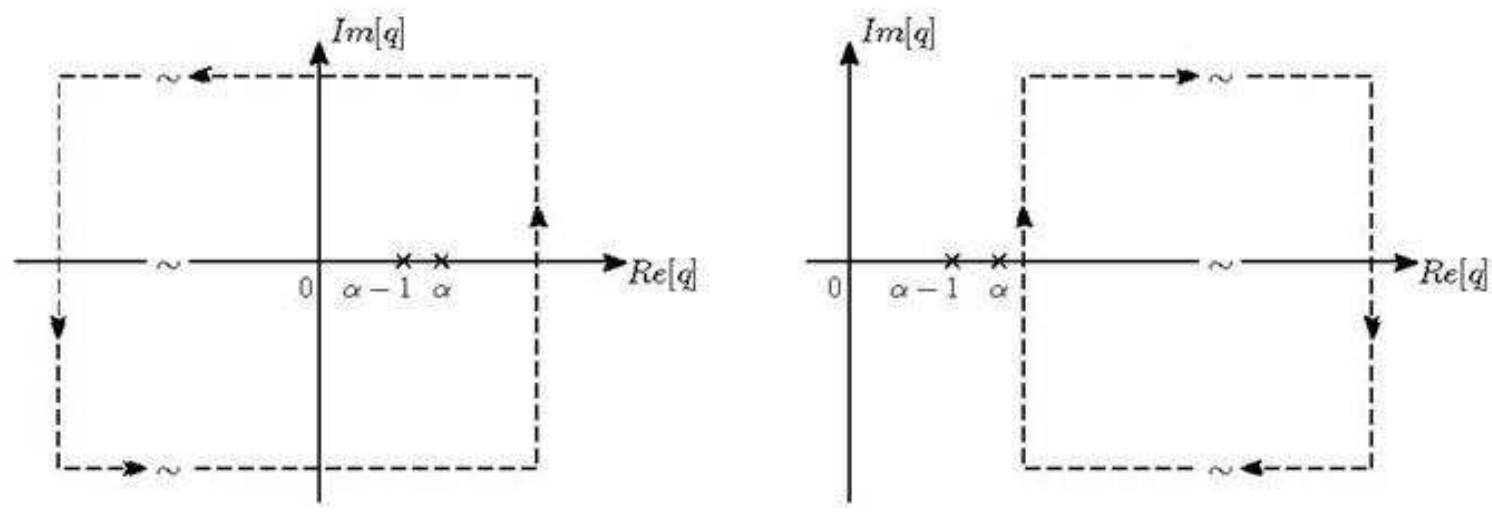

Figure 3. Contours for the calculation of the infinite density (ID) $\mathcal{I}_{\mathrm{TS}}(\bar{v})$, Eq. (4.9), of the two-state model, for $|\bar{v}|<v_{\mathrm{c}}$ (left panel) and $|\bar{v}|>v_{\mathrm{c}}$ (right panel). The poles $q=\alpha-1$ and $q=\alpha$ are found on $\operatorname{Re}(q)>0$ since $\alpha>1$. For general $F(v)$ the contour on the LHS panel may contain poles to the left of the $\operatorname{Re}(q)=0$ axis (if they exist).

For the two state model we obtain Eq. (4.10), where $F(v)$ does not have any residues. This can be extended to include a general discrete valued $\operatorname{PDF} F(v)=\sum_{j=1}^{N}\left[\delta\left(v-v_{c j}\right)+\delta\left(v+v_{c j}\right)\right] p_{j} / 2$, where $\left\{p_{j}\right\}_{j=1, \ldots, N}$ are probabilities, $\sum_{j=1}^{N} p_{j}=1$ and $v_{c j}>0$ for $j=1, \ldots, N$. Using Eqs. $(4.8,4.9,4.11)$ one finds the general form

$$
\mathcal{I}_{\mathrm{d}}(\bar{v})=\sum_{j=1}^{N} \frac{p_{j}}{v_{c j}} \mathcal{I}_{\mathcal{T S}}\left(\frac{\bar{v}}{v_{c j}}\right)
$$

Now we find the ID for a Gaussian test-case, $\mathcal{I}_{\mathrm{G}}(\bar{v})$, using Eq. (4.12). The velocity PDF is

$$
F_{\mathrm{G}}(v)=\frac{1}{\sqrt{2 \pi} V_{\mathrm{G}}} e^{-\frac{1}{2}\left(\frac{v}{V_{\mathrm{G}}}\right)^{2}},
$$

with

$$
\left\langle\left|v^{q}\right|\right\rangle=\frac{\left(\sqrt{2} V_{\mathrm{G}}\right)^{q}}{\sqrt{\pi}} \Gamma\left(\frac{1+q}{2}\right) .
$$

Inserting the above to the upper left parenthesis of Eq. (4.12) we find

$$
\frac{(\alpha-1)\left\langle|v|^{\alpha-1}\right\rangle}{\alpha\left\langle|v|^{\alpha}\right\rangle}=\frac{(\alpha-1) 2^{-\frac{1}{2}} \Gamma(\alpha / 2)}{\alpha \Gamma(1 / 2+\alpha / 2) V_{\mathrm{G}}}=\frac{2^{\frac{1}{2}} \Gamma(\alpha / 2)}{\alpha \Gamma([\alpha-1] / 2) V_{\mathrm{G}}}
$$

We now turn to calculate the last term on the right hand side of Eq. (4.12). The residues of $\left\langle|v|^{q}\right\rangle$ are determined by $\Gamma\left[(1+q) / 2\right.$ ] [see Eq. (4.16)]. They are located at $q_{j}=-2 j-1$ for $j=0,1 \ldots$, which give the familiar singularities of the Gamma function. The residue value, for this single pole [4], is well known and easily evaluated by

$$
\operatorname{Res}\left(\left\langle|v|^{q}\right\rangle, q_{j}\right)=\frac{2(-1)^{j}}{\sqrt{\pi} \Gamma(1+j)\left(\sqrt{2} V_{\mathrm{G}}\right)^{2 j+1}} .
$$


Inserting this expression into the polynomial term in Eq. (4.12) yields

$$
\begin{gathered}
\frac{\mathcal{B}}{2} \sum_{j=0}^{\infty}\left[\frac{q_{j} \operatorname{Res}\left(\left\langle|v|^{q}\right\rangle, q_{j}\right)}{\left(q_{j}-\alpha\right)\left(q_{j}-\alpha+1\right)}|\bar{v}|^{-q_{j}-1}\right]_{q_{j}=-2 j-1}=-\frac{\mathcal{B}}{2} \sum_{j=0}^{\infty} \frac{(2 j+1)}{(2 j+1+\alpha)(2 j+\alpha)} \frac{\left(\sqrt{2} V_{\mathrm{G}}\right)^{-2 j-1}}{\sqrt{\pi}} \frac{2(-1)^{j}}{\Gamma(1+j)}|\bar{v}|^{2 j} \\
=-\frac{\mathcal{B}}{2 \sqrt{2 \pi} V_{\mathrm{G}}} \sum_{j=0}^{\infty} \frac{(j+1 / 2)}{(j+[1+\alpha] / 2)(j+\alpha / 2) \Gamma(1+j)}\left[-\frac{\bar{v}^{2}}{2\left(V_{\mathrm{G}}\right)^{2}}\right]^{j} \\
=-\frac{\mathcal{B}}{2 \sqrt{2 \pi} V_{\mathrm{G}}} \sum_{j=0}^{\infty} \frac{\Gamma(j+3 / 2) \Gamma(j+[1+\alpha] / 2) \Gamma(j+\alpha / 2)}{\Gamma(j+1 / 2) \Gamma(j+[3+\alpha] / 2) \Gamma(j+1+\alpha / 2)} \frac{1}{\Gamma(1+j)}\left[-\frac{\bar{v}^{2}}{2\left(V_{\mathrm{G}}\right)^{2}}\right]^{j} .
\end{gathered}
$$

Recalling the definition of the Pochhammer symbol [4]

$$
\begin{aligned}
- & \frac{\mathcal{B}}{2 \sqrt{2 \pi} V_{\mathrm{G}}} \frac{\Gamma(3 / 2) \Gamma([1+\alpha] / 2) \Gamma(\alpha / 2)}{\Gamma(1 / 2) \Gamma([3+\alpha] / 2) \Gamma(1+\alpha / 2)} \sum_{n=0}^{\infty} \frac{(3 / 2)_{n}([1+\alpha] / 2)_{n}(\alpha / 2)_{n}}{(1 / 2)_{n}([3+\alpha] / 2)_{n}(1+\alpha / 2)_{n}} \frac{1}{n !}\left|-\frac{\bar{v}^{2}}{2\left(V_{\mathrm{G}}\right)^{2}}\right|^{n} \\
& =-\frac{1}{\sqrt{2 \pi}} \frac{\mathcal{B}}{\alpha(\alpha+1) V_{\mathrm{G}}}{ }_{3} F_{3}\left(\frac{3}{2}, \frac{1+\alpha}{2}, \frac{\alpha}{2} ; \frac{1}{2}, 1+\frac{\alpha}{2}, \frac{3+\alpha}{2} ;-\frac{\bar{v}^{2}}{2\left(V_{\mathrm{G}}\right)^{2}}\right),
\end{aligned}
$$

where ${ }_{3} F_{3}(\cdot)$ is a Hypergeometric function [4]. Combining Eqs. (4.17) and (4.20) yields

$\mathcal{I}_{\mathrm{G}}(\bar{v})=\frac{\alpha(\sqrt{2})^{\alpha} \Gamma\left(\frac{1+\alpha}{2}\right) \mathcal{B}}{2 \sqrt{\pi}} \frac{\left(V_{\mathrm{G}}\right)^{\alpha}}{|\bar{v}|^{\alpha+1}}\left(1-\frac{2^{\frac{1}{2}} \Gamma\left(\frac{\alpha}{2}\right)}{\alpha \Gamma\left(\frac{\alpha-1}{2}\right)} \frac{|\bar{v}|}{V_{\mathrm{G}}}\right)-\frac{1}{\sqrt{2 \pi}} \frac{\mathcal{B}}{\alpha(\alpha+1) V_{\mathrm{G}}}{ }_{3} F_{3}\left(\frac{3}{2}, \frac{\alpha}{2}, \frac{1+\alpha}{2} ; 1+\frac{\alpha}{2}, \frac{3+\alpha}{2}, \frac{1}{2} ;-\frac{1}{2}\left|\frac{\bar{v}}{V_{\mathrm{G}}}\right|^{2}\right)$

The same result was found in [41] using a characteristic function method and plotted there.

As noted in $[41,42]$, the infinite density is non-normalizable and yields in the long time limit the correct moments of order larger than $\alpha$ in accordance with its strip of definition. One may ask what is the relation with the PDF $P(x, t)$ which yields these moments exactly at all times. The answer is found in Eq. (4.7). The right hand side of this equation is exactly $t^{\alpha} P(x, t)$. Thus we find

$$
\mathcal{I}_{\mathrm{d}}\left(\frac{x}{t}\right) \sim t^{\alpha} P(x, t)
$$

for $t \rightarrow \infty$ and $x \neq 0$. Obtaining the density $P(x, t)$ as a measured result from an experiment allows the extraction of the ID. The latter a physical reality containing information on the particles' position and the moments. Eq. (4.22) also makes it clear why the ID is non-normalizable at $t \rightarrow \infty$.

\subsection{The Mellin convolution approach}

The inverse Mellin form of the ID, Eq. (4.8), and the three components of Eq. (4.12), are complicated to calculate. The first requires to perform complex integrals and the latter's exact form requires calculating all the residues. We present a more familiar and intuitive convolution form. The Mellin convolution is a multiplicative convolution [38], also known in statistics as a method of calculating the PDF of a product of two independent random variables.

We define for $\bar{v}>0$ the functions $B_{1}(\bar{v})$ and $B_{2}(\bar{v})$ with the following Mellin transforms $M_{1}(q)$ and $M_{2}(q)$ respectively. The strips of definitions are given by $q \in \mathbb{S}_{1}$ and $q \in \mathbb{S}_{2}$. The multiplicative convolution is defined as

$$
B_{\text {conv }}(\bar{v})=\int_{0}^{\infty} B_{1}(y) B_{2}\left(\frac{\bar{v}}{y}\right) \frac{d y}{y}
$$

an associative operation [38]. One can show that the Mellin transform of the convolution is

$$
\int_{0}^{\infty} \bar{v}^{q} B_{\mathrm{conv}}(\bar{v}) d \bar{v}=M_{1}(q+1) M_{2}(q+1)
$$

with the strip of definition $q \in \mathbb{S}_{1} \cap \mathbb{S}_{2}$. Specifically, we set $B_{\text {conv }}(\bar{v})=\mathcal{I}_{\mathrm{d}}(\bar{v})$ and $\left\langle|x(t)|^{q}\right\rangle /\left(2 t^{q-\alpha+1}\right)$, Eq. (4.5), as the convolution's product of Eq. (4.24). Expressing the latter as Eq. (4.4), we multiply and 
divide the by $\left(v_{\mathrm{c}}\right)^{q}$, where $v_{\mathrm{c}}$ is an arbitrary velocity constant. The resulting function can be partitioned into two components

$$
\left\{\begin{array}{l}
M_{1}(q+1)=\frac{\mathcal{B}}{2} \frac{q}{(q-\alpha)(q-\alpha+1)}\left(v_{\mathrm{c}}\right)^{q} \\
M_{2}(q+1)=\left\langle\left|\frac{v}{v_{\mathrm{c}}}\right|^{q}\right\rangle .
\end{array}\right.
$$

The inverse Mellin transform of $M_{1}(q+1)$ is $B_{1}(\bar{v})=\mathcal{I}_{\mathrm{TS}, v_{\mathrm{c}}}(\bar{v})$ which by using Eq. (4.9) can also be written as

$$
B_{1}(\bar{v})=\frac{1}{v_{\mathrm{c}}} \mathcal{I}_{\mathcal{T S}}\left(\frac{\bar{v}}{v_{\mathrm{c}}}\right)
$$

and for $M_{2}(q+1)$

$$
B_{2}(y)=2 v_{\mathrm{c}} F\left(v_{\mathrm{c}} y\right)
$$

where $y$ is a unit-less variable. Notice that $\mathcal{I}_{\mathrm{TS}, 1}(\cdot)$ is a unit-less function with a unit-less input variable. Changing $y$ to $v=y v_{\mathrm{c}}$ and inserting both functions to Eq. (4.23) results in

$$
\mathcal{I}_{\mathrm{d}}(\bar{v})=2 \int_{0}^{\infty} F(v) \mathcal{I}_{\mathcal{T S}}\left(\frac{\bar{v}}{v}\right) \frac{\mathrm{d} v}{v}
$$

This can be written with the aid of Eq. (4.10)

$$
\mathcal{I}_{\mathrm{d}}(\bar{v})=\mathcal{B}\left[\frac{\alpha \mathcal{F}_{\alpha}(\bar{v})}{|\bar{v}|^{1+\alpha}}-\frac{(\alpha-1) \mathcal{F}_{\alpha-1}(\bar{v})}{|\bar{v}|^{\alpha}}\right],
$$

where

$$
\mathcal{F}_{\alpha}(\bar{v})=\int_{|\bar{v}|}^{\infty} v^{\alpha} F(v) \mathrm{d} v .
$$

Eq. (4.29) for the ID $\mathcal{I}_{\mathrm{d}}(\bar{v})$ is simpler than the expression in the previous section, merely including two simple integrals, the integrand is a product of $F(v)$ and $v^{\alpha}$ or $v^{\alpha-1}$.

In [42] Eq. (4.29) was derived based on entirely different approach, and it was used to calculate the ID for the Gaussian $F_{\mathrm{G}}(v)$, Eq. (4.15).

$$
\mathcal{I}_{\mathrm{G}}(\bar{v})=\frac{\mathcal{B}}{2 \sqrt{2 \pi} V_{\mathrm{G}}}\left[\alpha \Gamma\left(\frac{1+\alpha}{2}, \frac{1}{2}\left|\frac{\bar{v}}{V_{\mathrm{G}}}\right|^{2}\right)\left|\frac{\sqrt{2} V_{\mathrm{G}}}{\bar{v}}\right|^{1+\alpha}-(\alpha-1) \Gamma\left(\frac{\alpha}{2}, \frac{1}{2}\left|\frac{\bar{v}}{V_{\mathrm{G}}}\right|^{2}\right)\left|\frac{\sqrt{2} V_{\mathrm{G}}}{\bar{v}}\right|^{\alpha}\right]
$$

where $\Gamma(\alpha, y)=\int_{y}^{\infty} e^{-t} t^{\alpha-1} \mathrm{~d} t$ is the incomplete Gamma function. This expression is equivalent to Eq. (4.21).

The $\mathcal{I}_{\mathrm{d}}(\bar{v})$, Eq. (4.28), can be attributed a statistical meaning as a product distribution [22]. Interpreting it probabilistically we find that $\mathcal{I}_{\mathcal{T} \mathcal{S}}(\bar{\nu})$ is the distribution of $\bar{\nu}=\left(T_{+}-T_{-}\right) / t$, where $T_{ \pm}$is the total time the particle moved in the \pm direction respectively. To see this simply note that in the two state model with $v_{c}=1$ the time-averaged velocity is exactly $\left(T_{+}-T_{-}\right) / t$. For a general process, the velocity $\operatorname{PDF} F(v)$ must be taken into account yielding that $\bar{v}=v\left(T_{+}-T_{-}\right) / t$, which describes the extreme excursions of this regime's ballistic nature.

\subsection{The infinite density tail}

The infinite density describes the density packet extremities at $x \sim t$. We now show that in the limit of large $\bar{v}$

$$
\mathcal{I}_{\mathrm{d}}(\bar{v}) \sim \mathcal{B} \frac{1-Q(|\bar{v}|)}{|\bar{v}|}
$$


where $Q(\bar{v})=\int_{-\infty}^{\bar{v}} F(v) \mathrm{d} v$ is the velocity cumulative distribution function (CDF). To demonstrate Eq. (4.32) we integrate Eq. (4.30) by parts yielding

$$
\mathcal{F}_{\alpha}(\bar{v})=|\bar{v}|^{\alpha}[1-Q(|\bar{v}|)]+\alpha \int_{|\bar{v}|}^{\infty} v^{\alpha-1}[1-Q(v)] \mathrm{d} v,
$$

We use L'Hôpital's rule to show that the first element in the RHS is the dominant one.

$$
\lim _{\bar{v} \rightarrow \infty} \frac{\bar{v}^{\alpha}[1-Q(\bar{v})]}{\alpha \int_{0}^{\bar{v}} v^{\alpha-1}[1-Q(v)] \mathrm{d} v}=\lim _{\bar{v} \rightarrow \infty} \frac{\alpha \bar{v}^{\alpha-1}[1-Q(\bar{v})]-\bar{v}^{\alpha} F(\bar{v})}{\alpha \bar{v}^{\alpha-1}[1-Q(\bar{v})]}=1-\frac{1}{\alpha} \lim _{\bar{v} \rightarrow \infty} \frac{\bar{v} F(\bar{v})}{1-Q(\bar{v})} .
$$

Applying L'Hôpital's rule a second time,

$$
\frac{1}{\alpha} \lim _{\bar{v} \rightarrow \infty} \frac{-\bar{v} F(\bar{v})}{1-Q(\bar{v})} \propto \lim _{\bar{v} \rightarrow \infty} \frac{\bar{v}}{F(\bar{v})} \frac{\mathrm{d} F(\bar{v})}{\mathrm{d} \bar{v}} \rightarrow \infty .
$$

Here we used the fact that all the positive ordered moments of $F(v)$ are finite, meaning, $\lim _{\bar{v} \rightarrow \infty} \bar{v}^{\zeta} F(v) \rightarrow$ 0 for $\zeta \geq 0$. Acknowledging that $\mathcal{F}_{\alpha}(\bar{v}) \sim|\bar{v}|^{\alpha}[1-Q(|\bar{v}|)]$ for large $|\bar{v}|$ and applying to Eq. (4.29) we find Eq. (4.32).

Calculating moments of order $q \gg \alpha$ we can use Eq. (4.32) since the far tail's contribution is more dominant. Putting that to the test by inserting Eq. (4.32) into Eq. (4.5) one finds after integration by parts

$$
\left\langle|x(t)|^{q}\right\rangle \sim 2 \mathcal{B} t^{q+1-\alpha} \int_{0}^{\infty} \frac{1-Q(|\bar{v}|)}{|\bar{v}|} \bar{v}^{q} \mathrm{~d} \bar{v}=\mathcal{B} \frac{1}{q}\left\langle|v|^{q}\right\rangle t^{q+1-\alpha} .
$$

This is the same as Eq. (4.4) in the limit $q \gg \alpha$

\subsection{The relation between the velocity and jump models}

The ID for the jump model can be derived using the same methods of Sec. (4.1) and $\left\langle\tilde{x}^{m}(t)\right\rangle$, Eq. (3.15). Probably the simplest way, though, is to notice the relation between Eqs. (3.10) and (3.15) in the long time limit,

$$
\left\langle x^{m}(t)\right\rangle \sim \frac{m}{\alpha}\left\langle\tilde{x}^{m}(t)\right\rangle,
$$

for even $m=2,4, \ldots$. We assumed in Sec. 4.1 that $\left\langle x^{m}(t)\right\rangle$ can be analytically continued as $\left\langle|x(t)|^{q}\right\rangle$, Eq. (4.4), for $q>\alpha$. The same goes for the jump model as we extend the validation of Eq. (3.15) to the absolute value moments of any order $q>\alpha$,

$$
\left\langle\left|\tilde{x}^{q}(t)\right|\right\rangle \sim \mathcal{B} \frac{\alpha\left\langle|v|^{q}\right\rangle}{(q-\alpha)(q-\alpha+1)} t^{q+1-\alpha} .
$$

The relation between the two models is summarized as

$$
\left\langle|x(t)|^{q}\right\rangle \sim \frac{q}{\alpha}\left\langle\left|\tilde{x}^{q}(t)\right|\right\rangle
$$

Dividing by $t^{q+1-\alpha}$ and inserting into Eq. (4.7) we find a relation between the IDs of the jump and the velocity models

$$
\mathcal{I}_{\mathrm{d}}(\bar{v})=-\frac{1}{\alpha} \frac{\mathrm{d}}{\mathrm{d} \bar{v}}\left[\bar{v} \tilde{\mathcal{I}}_{\mathrm{d}}(\bar{v})\right] .
$$

Clearly $\tilde{\mathcal{I}}_{\mathrm{d}}(\bar{v})$ is the corresponding ID of the jump model analyzed with the velocity PDF, $F(v)$. Integrating from $|\bar{v}|$ to $\infty$ we find

$$
\tilde{\mathcal{I}}_{\mathrm{d}}(\bar{v})=\frac{\alpha}{|\bar{v}|} \int_{|\bar{v}|}^{\infty} \mathcal{I}_{\mathrm{d}}(v) \mathrm{d} v
$$


For the jump model we apply Eq. (4.41) to Eq. (4.8),

$$
\tilde{\mathcal{I}}_{\mathrm{d}}(\bar{v})=\frac{\mathcal{B}}{4 \pi i} \int_{c-i \infty}^{c+i \infty} \frac{\alpha}{(q-\alpha)(q-\alpha+1)} \frac{\left\langle|v|^{q}\right\rangle}{|\bar{v}|^{q+1}} \mathrm{~d} q .
$$

The counterpart of Eq. (4.12) is given by

$$
\begin{aligned}
\tilde{\mathcal{I}}_{\mathrm{d}}(\bar{v}) & =\frac{\alpha \mathcal{B}}{2} \frac{\left\langle|v|^{\alpha}\right\rangle}{|\bar{v}|^{1+\alpha}}\left(1-\frac{\left\langle|v|^{\alpha-1}\right\rangle}{\left\langle|v|^{\alpha}\right\rangle}|\bar{v}|\right) \\
& +\frac{\alpha \mathcal{B}}{2} \sum_{j=1}^{n_{q}}\left[\frac{\operatorname{Res}\left(\left\langle|v|^{q_{j}}\right\rangle, q_{j}\right)}{\left(q_{j}-\alpha\right)\left(q_{j}-\alpha+1\right)}|\bar{v}|^{-q_{j}-1}\right] .
\end{aligned}
$$

Finally, the counterpart of Eq. (4.29) is found by inserting it into Eq. (4.41),

$$
\tilde{\mathcal{I}}_{\mathrm{d}}(\bar{v})=\alpha \mathcal{B}\left[\frac{\mathcal{F}_{\alpha}(\bar{v})}{|\bar{v}|^{1+\alpha}}-\frac{\mathcal{F}_{\alpha-1}(\bar{v})}{|\bar{v}|^{\alpha}} .\right]
$$

The simplest example is the two state process for the jump model, Eq. (4.10)

$$
\tilde{\mathcal{I}}_{\mathrm{TS}}(\bar{v})=\frac{\alpha \mathcal{B}}{2 v_{\mathrm{c}}}\left|\frac{v_{\mathrm{c}}}{\bar{v}}\right|^{\alpha+1}\left(1-\left|\frac{\bar{v}}{v_{\mathrm{c}}}\right|\right),
$$

for $0<|\bar{v}| \leq 1$ otherwise $\tilde{\mathcal{I}}_{\mathrm{TS}}(\bar{v})=0$. As discussed earlier, the particle is static during the sojourn times causing the ID to be zero at $|\bar{v}| / v_{c}=1$ (unlike the two state velocity model).

\section{The uniform approximation of $\mathrm{P}(x, t)$}

Our goal in this section is give an adequate approximation describing the Lévy walk propagator $P(x, t)$. Let us first discuss the typical fluctuations based on the central limit theorem arguments and Lévy statistics following [42]. The center of the packet is known to be described by the Lévy stable distribution function $[41,42]$. The predominating phenomenon is weakly correlated small jumps at short scales of $x$, more precisely when $x \sim t^{1 / \alpha}$ which is smaller than the ballistic scaling $x \sim t$. The result is the known Lévy central limit result [10,32], which takes effect in both the velocity and jump models. As we showed in $[42]$

$$
P_{\text {cen }}(k, u) \sim \frac{1}{u+K_{\alpha}|k|^{\alpha}} .
$$

This limit is found for the Montroll-Weiss equation assuming both $k$ and $u$ are both small and the ratio $k^{\alpha} / u$ is fixed. It thus describes $x$ scaling like $t^{1 / \alpha}$ which is out side the domain of the ballistic scaling of the tails. Inverse transforming the above in Fourier and Laplace yields

$$
P_{\text {cen }}(x, t)=\frac{1}{\left[K_{\alpha} t\right]^{\frac{1}{\alpha}}} L_{\alpha}\left(\frac{x}{\left[K_{\alpha} t\right]^{\frac{1}{\alpha}}}\right),
$$

where $K_{\alpha}$ is the anomalous diffusion coefficient [42] (see below). $L_{\alpha}(\cdot)$ is the symmetric Lévy stable PDF defined through Eq. (5.1).

For large $|x|, P_{\text {cen }}(x, t)$ is approximated by

$$
P_{\text {cen }}(x, t) \sim K_{\alpha} c_{\alpha} t|x|^{-1-\alpha},
$$

where we used $L_{\alpha}(x) \sim c_{\alpha}|x|^{-1-\alpha}$ and $c_{\alpha}=\Gamma(1+\alpha) \sin (\pi \alpha / 2) / \pi$. We compare $P_{\text {cen }}(x, t)$ for large $x$ with the IDs, Eqs. (4.12) and (4.43) at $\bar{v} \rightarrow 0$,

$$
\mathcal{I}_{\mathrm{d}}(\bar{v}) \sim \tilde{\mathcal{I}}_{\mathrm{d}}(\bar{v}) \sim \frac{\alpha\left\langle|v|^{\alpha}\right\rangle \mathcal{B}}{2}|\bar{v}|^{-1-\alpha}
$$


Using Eq. (4.22), we compare between Eqs. (5.3,5.4) and find an alternative form to Eq. (3.11),

$$
\mathcal{B}=\frac{2 K_{\alpha} c_{\alpha}}{\alpha\left\langle|v|^{\alpha}\right\rangle}
$$

Thus $K_{\alpha}$ can be expressed as [42]

$$
K_{\alpha}=\frac{A\left\langle|v|^{\alpha}\right\rangle}{\langle\tau\rangle}\left|\cos \frac{\pi \alpha}{2}\right|
$$

This matching between $P_{\text {cen }}(x, t)$ and $t^{-\alpha} \mathcal{I}_{\mathrm{d}}(x / t)$, the center and outer regions of the PDF, in the region $t^{\frac{1}{\alpha}} \ll|x| \ll t$ indicates that the two suffice to describe the process at the long time limit. See Fig. 4.

In [42] we established that the Lévy distribution, Eq. (5.2) and the infinite density Eq. (4.22) complement each other in the long time limit. The first is the sole contributor to moments of order less than $\alpha$ and the latter is used for calculation of those of order larger than $\alpha$. Both must be used to describe the particle density $P(x, t)$. Using the fact that the power law form, Eq. (5.3), is shared by both densities in the intermediate range we determine the uniform approximation of $P(x, t)$, combining the center, intermediate and outer regions. The approximation must be normalizable and gives the correct moments at $t \rightarrow \infty$. Using Eqs. (4.22), (5.2) and (5.3) we find

$$
\begin{aligned}
P(x, t) & \sim \frac{\frac{1}{\left(K_{\alpha} t\right)^{1 / \alpha}} L_{\alpha}\left(\frac{x}{\left(K_{\alpha} t\right)^{1 / \alpha}}\right) t^{-\alpha} I_{d}\left(\frac{x}{t}\right)}{K_{\alpha} c_{\alpha} t|x|^{-1-\alpha}} \\
& =\frac{1}{c_{\alpha} t^{\alpha}}\left|\frac{x}{\left(K_{\alpha} t\right)^{1 / \alpha}}\right|^{1+\alpha} L_{\alpha}\left(\frac{x}{\left(K_{\alpha} t\right)^{1 / \alpha}}\right) I_{d}\left(\frac{x}{t}\right) .
\end{aligned}
$$

App. C deals with the moment calculation. For a numerical example see Fig. 5. From Eqs. $(4.4,5.2)$ it follows that for $1<\alpha<2$

$$
q \nu(q)=\left\{\begin{array}{r}
q / \alpha \text { for } q<\alpha \\
q+1-\alpha \text { for } q>\alpha
\end{array}\right.
$$

a behavior demonstrated with simulations in Fig. 1.

\section{Diffusive regimes: $\alpha>2$}

How general is the description of the packet of particles in terms of the infinite density? Is this nonnormalised density related to the Lévy density as shown in previous section, or can it be found also for models where the bulk PDFs are Gaussian? Above we addressed the sub-ballistic super diffusive regime case, $1<\alpha<2$, meaning that the flight time $\tau$ has a finite mean $\langle\tau\rangle$ but a diverging variance. We are now going to consider the Lévy walk when with the distribution $\psi(\tau)$ with $\alpha>2$, for which the variance is finite. Looking for its effect on $P_{\text {cen }}(x, t)$ and the infinite density, we follow the steps made in Secs. 2.1 and 3.1 .

\subsection{The generating function $\mathrm{P}(k, u)$}

As mentioned, the flight time $\operatorname{PDF} \psi(\tau)$, Eq. (2.2), has a power law tail and for $\alpha>2$ we have finite first and second moments. This indicates an expected relation between $P(x, t)$ and the Gaussian central limit theorem. Let $r \equiv\lfloor\alpha\rfloor$ and $r \geq 2$. According to a Tauberian theorem [51], the Laplace transform of $\hat{\psi}(u)$ has an asymptotic form in accordance with Eq. (2.3),

$$
\hat{\psi}(u) \sim 1+\sum_{j=1}^{r} \frac{(-1)^{j}}{j !}\left\langle\tau^{j}\right\rangle u^{j}+(-1)^{r+1} A u^{\alpha} \ldots,
$$

with $A>0$. Inserting this into the Montroll-Weiss equation, Eq. (2.6), and simplifying 


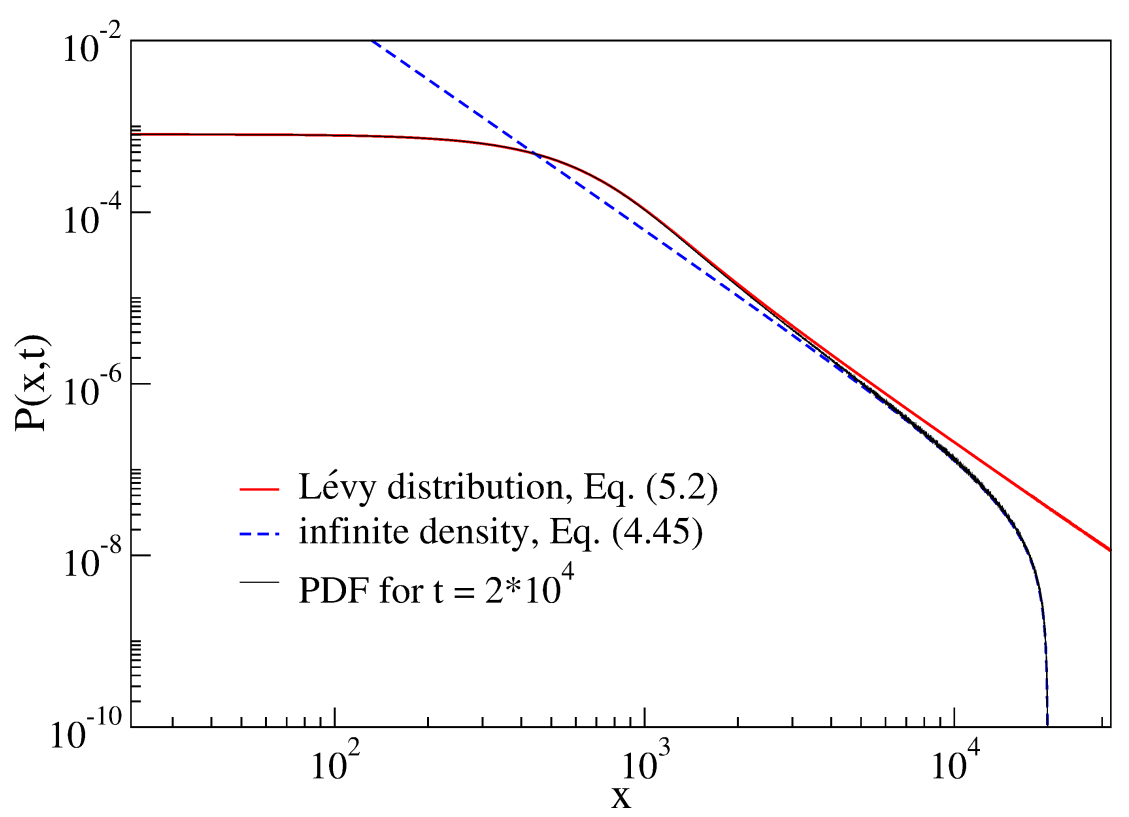

Figure 4. Two complementary densities of the jump model. For the central part of the density, $|x| \lesssim t^{1 / \alpha}$, the Lévy distribution (solid red line), Eq. (5.2), is a good approximation. The infinite density (dashed blue line), Eq. (4.45), nicely matches the tails of the packet of particles. The PDF of the process (thin black line) is barely visible due to the perfect agreement with theoretical predictions. The Lévy distribution and the ID curves overlap at the central region near $x \simeq 4000$. The PDF was sampled with $10^{10}$ realizations and measurement time was $2 * 10^{4}$.

$$
P(k, u) \sim \frac{\sum_{j=1}^{r} \frac{(-1)^{j}}{j !} \frac{\left\langle\tau^{j}\right\rangle}{\langle\tau\rangle}\left\langle[u-i k v]^{j-1}\right\rangle-(-1)^{r} A_{\tau}\left\langle[u-i k v]^{\alpha-1}\right\rangle}{\sum_{j=1}^{r} \frac{(-1)^{j}}{j !} \frac{\left\langle\tau^{j}\right\rangle}{\langle\tau\rangle}\left\langle[u-i k v]^{j}\right\rangle-(-1)^{r} A_{\tau}\left\langle[u-i k v]^{\alpha}\right\rangle},
$$

with $A_{\tau}=A /\langle\tau\rangle$. For future purposes we classify the nominator and the denominator of Eq. (6.2) as $\mathcal{N}(k, u)$ and $\mathcal{D}(k, u)$ respectively. To investigate $P(x, t)$ in the bulk region $|x| \propto t^{1 / 2}$ and in the long time limit, we expand Eq. (6.2) for $|u| \ll|k v| \ll 1$ using the symmetry of $F(v)$. We find the Fourier-Laplace transform of $P(x, t)$ describing the center part of the packet of particles

$$
P_{\text {cen }}(k, u) \sim \frac{1}{u+\frac{\left\langle\tau^{2}\right\rangle}{2\langle\tau\rangle}\left\langle v^{2}\right\rangle k^{2}} .
$$

Its inverse Fourier-Laplace transform is a Gaussian PDF with zero mean and a variance

$$
\left\langle x^{2}\right\rangle=\left\langle v^{2}\right\rangle\left\langle\tau^{2}\right\rangle \frac{t}{\langle\tau\rangle}
$$

The rest of the positive integer moments are derived in the following Section.

\subsection{Derivation of the integer moments}

We present the derivation of the moments for $r=\lfloor\alpha\rfloor, r \geq 2$. The derivation is in accordance with the one presented in Sec. 2.1 for the velocity model. For the final result turn to Eq. (6.18). The derivatives of the nominator, $\mathcal{N}(k, u)$, and denominator, $\mathcal{D}(k, u)$, are given for later use: 


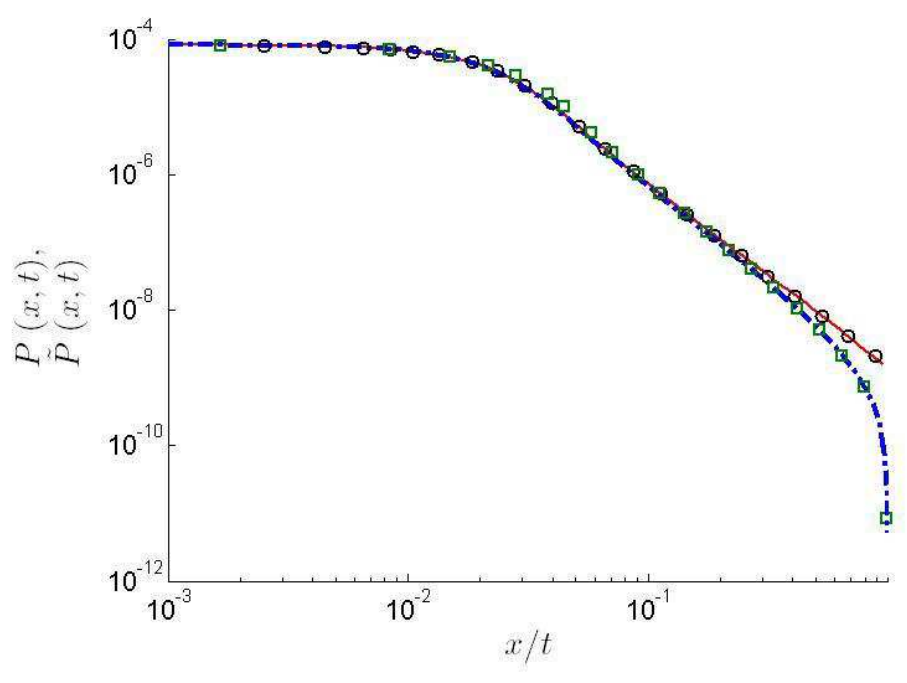

FiguRE 5. (color online) Uniform approximations (lines), Eq. (5.7), of the PDF for Lévy walks versus the numerically sampled PDFs for $t=2.5 \cdot 10^{5}$ (symbols). We use the two state velocity model (solid red line) and the jump (dashed-dotted blue line) model, Eq. (2.1), with $v_{\mathrm{c}}=1$. The parameters are $\alpha=1.5, A=|\Gamma(1-\alpha)|$. The PDFs were sampled over $10^{8}$ realizations.

$$
\left\{\begin{array}{l}
\left.\frac{\partial^{m}}{\partial(i k)^{m}} \mathcal{N}(k, u)\right|_{k=0}=\sum_{j=1}^{r} \frac{(-1)^{j-m}}{j} \frac{\left\langle\tau^{j}\right\rangle}{\langle\tau\rangle}\left\langle v^{m}\right\rangle \frac{1}{\Gamma(j-m)} u^{j-m-1}-(-1)^{m+r} A_{\tau}\left\langle v^{m}\right\rangle \frac{\Gamma(\alpha)}{\Gamma(\alpha-m)} u^{\alpha-m-1}, \\
\left.\frac{\partial^{m}}{\partial(i k)^{m}} \mathcal{D}(k, u)\right|_{k=0}=\sum_{j=1}^{r}(-1)^{j-m} \frac{\left\langle\tau^{j}\right\rangle}{\langle\tau\rangle}\left\langle v^{m}\right\rangle \frac{1}{\Gamma(j-m+1)} u^{j-m}-(-1)^{m+r} A_{\tau}\left\langle v^{m}\right\rangle \frac{\Gamma(\alpha+1)}{\Gamma(\alpha+1-m)} u^{\alpha-m} .
\end{array}\right.
$$

The moments $\left\langle x^{m}(u)\right\rangle$ are given by the general Leibniz rule of derivation:

$$
\left\langle x^{m}(u)\right\rangle=\left.\frac{\partial^{m}}{\partial(i k)^{m}} P(k, u)\right|_{k=0}=\left.\sum_{j=0}^{m}\left(\begin{array}{c}
m \\
j
\end{array}\right) \frac{\partial^{m-j}}{\partial(i k)^{m-j}} \mathcal{N}(k, u) \frac{\partial^{j}}{\partial(i k)^{j}} \frac{1}{\mathcal{D}(k, u)}\right|_{k=0} .
$$

The exact expression for the derivative of the denominator is given by the Faà di Bruno formula [33]

$$
\left.\frac{\partial^{j}}{\partial(i k)^{j}} \frac{1}{\mathcal{D}(k, u)}\right|_{k=0}=\sum_{z=1}^{j} \frac{(-1)^{z} z !}{\mathcal{D}(k=0, u)^{z+1}} B_{j, z}\left(\omega_{1}, \omega_{2}, \ldots, \omega_{j-z+1}\right),
$$

where $B_{j, z}\left(\omega_{1}, \omega_{2}, \ldots, \omega_{j-z+1}\right)$ is the partial Bell polynomial, Eq. (3.4), and the summation is taken over all the sequences of integers $r_{1}, r_{2}, \ldots \geq 0$ such that :

$$
\left\{\begin{array}{l}
r_{2}+r_{4}+\ldots=z \\
2 r_{2}+4 r_{4} \ldots=j
\end{array}\right.
$$

The odd indices are $r_{j}=0$ due to the symmetry of velocity PDF, $F(v)$. Thus $j$ must be even, meaning

$$
\left\{\begin{array}{r}
r_{2}+r_{4}+\ldots=z \\
r_{2}+2 r_{4} \ldots=j / 2
\end{array}\right.
$$


where the maximum possible value which $z$ and $r_{2}$ attain is $j / 2$. In the small $u$ limit, the inputs $\left\{\omega_{z}\right\}_{z=1,2, \ldots}$ of $B_{j, z}(\cdot)$ are

$$
\omega_{z} \sim\left\{\begin{array}{r}
0 \text { odd } z \\
-\frac{\left\langle\tau^{z}\right\rangle}{\langle\tau\rangle}\left\langle v^{z}\right\rangle \quad z \leq r \\
-A_{\tau}(-\alpha)_{z}\left\langle v^{z}\right\rangle u^{\alpha-z} \quad r<z .
\end{array}\right.
$$

We now find the long time behavior of the moments, Eq. (6.6), corresponding to the small $u$ limit. For $m$, an even order of the moment, we differentiate between $m<\alpha$ and $\alpha<m$ for convenience. The first case is $0<m<\alpha$ :

$$
\begin{aligned}
\left\langle x^{m}(u)\right\rangle & =\left.\frac{1}{\mathcal{D}(k, u)} \frac{\partial^{m} \mathcal{N}(k, u)}{\partial(i k)^{m}}\right|_{k=0}+\left.\left.\sum_{j=1}^{m}\left(\begin{array}{c}
m \\
j
\end{array}\right) \frac{\partial^{m-j} \mathcal{N}(k, u)}{\partial(i k)^{m-j}}\right|_{k=0} \frac{\partial^{j}}{\partial(i k)^{j}} \frac{1}{\mathcal{D}(k, u)}\right|_{k=0} \\
& \sim \frac{1}{u}\left[\frac{\left\langle\tau^{m+1}\right\rangle\left\langle v^{m}\right\rangle}{\langle\tau\rangle(m+1)} I(m<r)+(-1)^{r+m} A_{\tau}\left\langle v^{r}\right\rangle \frac{\Gamma(\alpha)}{\Gamma(\alpha-r)} u^{\alpha-1-r} \delta_{r, m}\right] \\
& +\sum_{j=1}^{m}\left(\begin{array}{c}
m \\
j
\end{array}\right)\left[\frac{\left\langle\tau^{m-j+1}\right\rangle\left\langle v^{m-j}\right\rangle}{\langle\tau\rangle(m-j+1)} I(m-j<r)+(-1)^{r+m-j} A_{\tau}\left\langle v^{r}\right\rangle \frac{\Gamma(\alpha)}{\Gamma(\alpha-r)} u^{\alpha-1-r} \delta_{r, m-j}\right] \sum_{z=1}^{j} \frac{(-1)^{z} z !}{u^{z+1}} B
\end{aligned}
$$

where $I(\cdots)=1$ if the statement in the parenthesis is true and zero otherwise while $\delta_{r, m}=1$ if $r=m$ otherwise it is 0 . Since $j \leq m \leq r$ then Eq. (6.10) yields $B_{j, z}$ a constant independent of $u$. Hence the minimum exponent of $u^{-1-z} B_{j, z}$ in the last term in Eq. (6.14) is given for $r_{2}=z=j / 2$, where

$$
B_{j, j / 2}\left(0, \omega_{2}, 0, \ldots\right)=\frac{j !}{(j / 2) !}\left(\frac{\omega_{2}}{2}\right)^{\frac{j}{2}} .
$$

In the long time limit the asymptotic form of the moment is given by

$$
\left\langle x^{m}(u)\right\rangle \sim\left(\frac{\left\langle v^{2}\right\rangle\left\langle\tau^{2}\right\rangle}{2\langle\tau\rangle}\right)^{\frac{m}{2}} \frac{m !}{u^{1+\frac{m}{2}}}+\frac{\Gamma(\alpha)}{\Gamma(\alpha-r)} \frac{A_{\tau}\left\langle v^{r}\right\rangle}{u^{r+2-\alpha}} \delta_{r, m} .
$$

Performing the inverse Laplace transform on the latter we find for the dominant order

$$
\left\langle x^{m}(t)\right\rangle \sim \frac{\Gamma(m+1)}{\Gamma\left(\frac{m}{2}+1\right)}\left(\frac{\left\langle v^{2}\right\rangle\left\langle\tau^{2}\right\rangle}{2\langle\tau\rangle} t\right)^{\frac{m}{2}} .
$$

These are the moments of the Gaussian distribution in accordance with $P_{\text {cen }}(k, u)$, Eq. (6.3).

Turning our attention to even moments of order $m>\alpha$, they are expressed by

$$
\begin{aligned}
\left\langle x^{m}(u)\right\rangle & =\left.\frac{1}{\mathcal{D}(k, u)} \frac{\partial^{m} \mathcal{N}(k, u)}{\partial(i k)^{m}}\right|_{k=0}+\left.\left[\sum_{j=1}^{r^{*}}+\sum_{j=r^{*}+1}^{m}\right]\left(\begin{array}{c}
m \\
j
\end{array}\right) \frac{\partial^{m-j} \mathcal{N}(k, u)}{\partial(i k)^{m-j}} \frac{\partial^{j}}{\partial(i k)^{j}} \frac{1}{\mathcal{D}(k, u)}\right|_{k=0} \\
& \sim \frac{1}{u}(-1)^{r+m} A_{\tau}\left\langle v^{m}\right\rangle \frac{\Gamma(\alpha)}{\Gamma(\alpha-m)} u^{\alpha-1-m}+\sum_{j=1}^{r^{*}}[\ldots]+\sum_{j=r^{*}+1}^{m}\left(\begin{array}{c}
m \\
j
\end{array}\right) \frac{\left\langle\tau^{m-j+1}\right\rangle\left\langle v^{m-j}\right\rangle}{\langle\tau\rangle(m-j+1)} \sum_{z=1}^{j} \frac{(-1)^{z} z !}{u^{z+1}} B_{j, z}(\omega
\end{aligned}
$$

where $r^{*}$ is large enough such that $m-r^{*}<r$ and the leading order of the nominator's derivative is a constant $u^{0}$. After careful analysis one finds that the dominant contributions are brought by the first term from the left and the sum, $\sum_{j=r^{*}+1}^{m}$. The latter contains the denominator's derivative whose leading term varies between

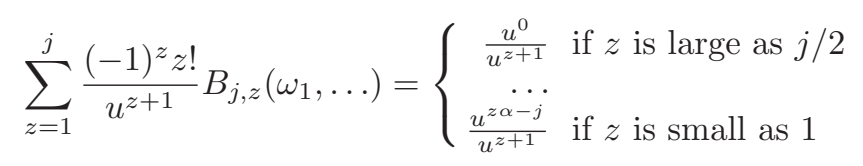


Performing the calculations we find the leading contributions corresponding to $j=m$ with $z=1$ and $j / 2$.

$$
\left\langle x^{m}(u)\right\rangle \sim m !\left(\frac{\left\langle v^{2}\right\rangle\left\langle\tau^{2}\right\rangle}{2\langle\tau\rangle}\right)^{\frac{m}{2}} \frac{1}{u^{\frac{m}{2}+1}}+\left[\frac{\Gamma(\alpha)}{\Gamma(\alpha-m)}-\frac{\Gamma(\alpha+1)}{\Gamma(\alpha+1-m)}\right] \frac{(-1)^{n+m} A_{\tau}\left\langle v^{m}\right\rangle}{u^{m+2-\alpha}} .
$$

Using the inverse Laplace transform and Gamma function identities [4] we find the expression for moments of even order $m>\alpha$

$$
\left\langle x^{m}(t)\right\rangle \simeq \frac{\Gamma(m+1)}{\Gamma\left(\frac{m}{2}+1\right)}\left(\frac{\left\langle v^{2}\right\rangle\left\langle\tau^{2}\right\rangle}{2\langle\tau\rangle} t\right)^{\frac{m}{2}}+\mathcal{B} \frac{m\left\langle v^{m}\right\rangle}{(m-\alpha)(m-\alpha+1)} t^{m+1-\alpha} .
$$

Thus we find two terms the first grows like $t^{m / 2}$ and it describes Gaussian statistics, i.e. moments of the normal distribution, and a second term proportional to $t^{m+1-\alpha}$, which originates from the tail of $P(x, t)$. Importantly the latter is similar to the expression in Eq. (3.10) for $1<\alpha<2$. This means that the ID for $\alpha>2$ is the same as that found for $1<\alpha<2$, since the ID must generate the moments of the process. To see this notice that the second term in Eq. (6.18) is larger than the first, in the long time limit, when $m>2(\alpha-1)$. So the $q$ moments larger than the critical value $2(\alpha-1)$ behave as for the super-diffusive phase $1<\alpha<2$ and the ID description of the tails of the PDF is valid for $\alpha>1$. Thus the critical value of $q$ after which the infinite density dominates is

$$
q_{c}(\alpha)=\left\{\begin{array}{lr}
\alpha & \text { if } 1<\alpha<2, \\
2(\alpha-1) & \text { if } 2<\alpha .
\end{array}\right.
$$

We note that it is difficult to either measure or simulate the rare events for $2<\alpha$ if compared with the case $\alpha \simeq 3 / 2$.

There is a difference between the Gaussian $2<\alpha$ and Lévy $1<\alpha<2$ phases specified next. The Lévy density and the ID match for intermediate $x$ as we have shown in the previous section. In contrast the Gaussian cannot be matched to the ID for intermediate values of $x$. To make the matching, one needs to find the correction to the central limit theorem, for example using the fractional Edgeworth expansion [26]. This could yield a power law correction term to the Gaussian which could in principle match the ID. This non universal correction leads to a non-universal behavior of the moments when $\alpha<m<2(\alpha-1)$ namely moments are not determined exclusively by either the Gaussian central limit theorem or the ID. We leave these issues to future rigorous work. The $q-\alpha$ phase diagram of the Lévy walk model is summarised in Fig. 6. It contains in it a stripe of non-universal features which is beyond the scope of this paper.

The above principles are true for the jump model as well. The infinite density is derived through the moments in the same manner as in Sec. 4, yielding the same results, Eqs.(4.8) and (4.42). All the former expressions found for the ID for $1<\alpha<2$ are valid for $\alpha>2$ such as Eqs. (4.10,4.12,4.28).

\section{Biased dynamics}

We mentioned in the Sec. 2 that the velocity $\mathrm{PDF}, F(v)$ is symmetric and assumed $\langle v\rangle=0$. We will now introduce bias by shifting the distribution

$$
F_{\langle v\rangle}(v)=F(v-\langle v\rangle) .
$$

This ensures that the moments $\left\langle[v-\langle v\rangle]^{q}\right\rangle=0$ for odd $q$. We introduce the $\operatorname{PDF}, P_{\langle v\rangle}(k, u)$, for the velocity model with the velocity $\operatorname{PDF}, F_{\langle v\rangle}(v)$, while for the unbiased case the $\operatorname{PDF} P(k, u)$ was given by Eq. (2.6), the Montroll-Weiss equation. By inserting Eq. (7.1) to the latter and changing the variables we find

$$
P_{\langle v\rangle}(k, u)=P(k, u-i k\langle v\rangle) .
$$




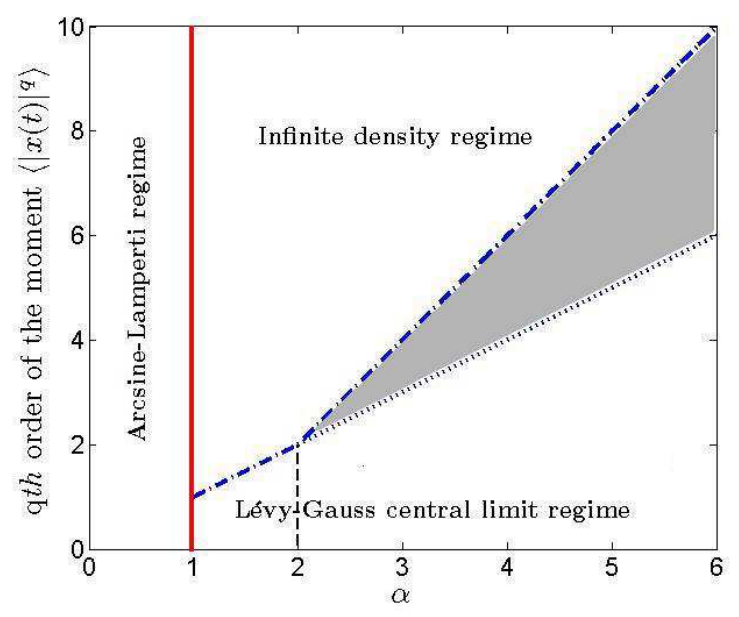

Figure 6. (color online) Phase diagram of the Lévy walk process on the parameter space $(q, \alpha)$. For $0<\alpha<1$ we find mono-scaling and ballistic transport, Eq. (8.3). For $q_{c}(\alpha)<q$, Eq. (6.19), the statistics is described by the infinite density (the region above the dot dashed line). Region $1<\alpha$ and $q<\alpha$ is governed by the Lévy-Gauss central limit theorem. The fourth region, marked grey, is not yet fully explored, since it requires non-trivial matching conditions between the ID and the Gauss central limit theorem.

This relation suits the jump model as well. We first perform the inverse Laplace transform and then inverse Fourier transform which yield

$$
P_{\langle v\rangle}(x, t)=P(x-\langle v\rangle t, t) .
$$

When a velocity bias is present in the system, one should measure $\langle x\rangle=\langle v\rangle t$. Then, one can use the same tools discussed earlier including the infinite density after transforming to the rest frame, $\frac{x}{t}-\langle v\rangle$. Then the relation between the biased case ID and the unbiased one is

$$
\mathcal{I}_{\mathrm{d},\langle v\rangle}(\bar{v})=\mathcal{I}_{\mathrm{d}}(\bar{v}-\langle v\rangle) .
$$

In Sec. $5, P_{\text {cen }}(x, t)$ was found to be a Lévy stable distribution for the case of $\langle v\rangle=0$. In the general case, the whole packet shifts to $x \sim\langle v\rangle t$ where the weakly correlated small jumps at relatively short times are dominant. Applying Eq. (7.2) to Eq. (5.1), followed by Fourier-Laplace transformation for $1<\alpha<2$ produces,

$$
P_{\text {cen },\langle v\rangle}(x, t)=\frac{1}{\left[K_{\alpha} t\right]^{\frac{1}{\alpha}}} L_{\alpha}\left(\frac{x-\langle v\rangle t}{\left[K_{\alpha} t\right]^{\frac{1}{\alpha}}}\right)
$$

\section{Ballistic regime: $0<\alpha<1$}

For $0<\alpha<1$ the motion becomes ballistic, namely, $x \sim t$. The propagators for the symmetric case were considered in Ref. [23] using methods from Refs. [25] and [39,40]. Below we obtain the PDF of the scaled variable $x / t$. We call this regime the ballistic Arcsine-Lamperti regime since as we show below the limit theorems describing the packet of particles are related to the work of Lamperti and the Arcsine law found by P. Lévy in the context of occupation times of Brownian motion (see Fig. 6). The problem at hand is related to the problem of ergodicity. To see this note that $x=\int_{0}^{t} v(t) \mathrm{d} t$ and hence the scaling variable $x / t$ is the time-average of the velocity. Since $0<\alpha<1$ the problem is related to weak ergodicity 
breaking $[39,40]$. To start the analysis we recall the Laplace transform of the flight times PDF in the small $u$ limit

$$
\hat{\psi}(u) \sim 1-A u^{\alpha} \ldots
$$

\subsection{The ballistic PDF}

For the remaining of the section we relax our constraints on $F(v)$, the velocity PDF, and allow any general form with all of its integer moments finite. For the velocity model we approximate the Montroll-Weiss equation, Eq. (2.6), in the small $k$ and $u$ limits such that $k / u$ is finite using Eq. (8.1),

$$
P(k, u) \sim \frac{\left\langle(u-i k v)^{\alpha-1}\right\rangle}{\left\langle(u-i k v)^{\alpha}\right\rangle}=\frac{1}{u} \frac{\left\langle\left(1-\frac{i k}{u} v\right)^{\alpha-1}\right\rangle}{\left\langle\left(1-\frac{i k}{u} v\right)^{\alpha}\right\rangle} .
$$

Seeking $P(x, t)$ in the long time limit we apply the tools discussed in [40], which refers to a FourierLaplace transform. The entire process is ballistic and dependent on the time-averaged velocity, $\bar{v}$, Eq. (4.6) [23]

$$
\begin{aligned}
\mathcal{P}(\bar{v}) & =\lim _{t \rightarrow \infty} t P(x, t) \\
& =-\frac{1}{\pi} \lim _{\epsilon \rightarrow 0} \operatorname{Im} \frac{\int_{-\infty}^{\infty} d v F(v)(\bar{v}-v+i \epsilon)^{\alpha-1}}{\int_{-\infty}^{\infty} d v F(v)(\bar{v}-v+i \epsilon)^{\alpha}} .
\end{aligned}
$$

Using the identities $\lim _{\epsilon \rightarrow 0}(\bar{v}-v+i \epsilon)^{\alpha}=|\bar{v}-v| e^{i \alpha \phi(v)}$ and $\phi(v)=\pi I(\bar{v}<v)$ we define

$$
H_{\alpha}^{<}(\bar{v})=\int_{\bar{v}}^{\infty} d v F(v)|\bar{v}-v|^{\alpha}
$$

and

$$
H_{\alpha}^{>}(\bar{v})=\int_{-\infty}^{\bar{v}} d v F(v)|\bar{v}-v|^{\alpha} .
$$

Inserting these into Eq. (8.3) gives the main result of this subsection,

$$
\mathcal{P}_{\alpha}(\bar{v})=\frac{\sin (\pi \alpha)}{\pi} \frac{H_{\alpha-1}^{<}(\bar{v}) H_{\alpha}^{>}(\bar{v})+H_{\alpha}^{<}(\bar{v}) H_{\alpha-1}^{>}(\bar{v})}{\left[H_{\alpha}^{<}(\bar{v})\right]^{2}+\left[H_{\alpha}^{>}(\bar{v})\right]^{2}+2 \cos (\pi \alpha) H_{\alpha}^{>}(\bar{v}) H_{\alpha}^{<}(\bar{v})} .
$$

For the jump model, we apply the long time limit approximation, Eq. (8.1), to the Montroll-Weiss equation, Eq. (2.8),

$$
\tilde{\mathcal{P}}(k, u) \sim \frac{u^{\alpha-1}}{\left\langle(u-i k v)^{\alpha}\right\rangle}=\frac{1}{u} \frac{1}{\left\langle\left(1-\frac{i k}{u} v\right)^{\alpha}\right\rangle} .
$$

Similarly, using [40] and the methods depicted above we find

$$
\tilde{\mathcal{P}}(\bar{v})=\lim _{t \rightarrow \infty} t \tilde{P}(x, t)=-\frac{|\bar{v}|^{\alpha-1}}{\pi} \lim _{\epsilon \rightarrow 0} \operatorname{Im} \frac{I(\bar{v}>0)-e^{i \pi \alpha} I(\bar{v}<0)}{\int_{-\infty}^{\infty} d v F(v)(\bar{v}-v+i \epsilon)^{\alpha}},
$$

or the second key function of this subsection,

$$
\tilde{\mathcal{P}}(\bar{v})=\frac{\sin (\pi \alpha)}{\pi}|\bar{v}|^{\alpha-1} \frac{H_{\alpha}^{<}(\bar{v}) I(\bar{v}>0)+H_{\alpha}^{>}(\bar{v}) I(\bar{v}<0)}{\left[H_{\alpha}^{<}(\bar{v})\right]^{2}+\left[H_{\alpha}^{>}(\bar{v})\right]^{2}+2 \cos (\pi \alpha) H_{\alpha}^{>}(\bar{v}) H_{\alpha}^{<}(\bar{v})} .
$$

$\mathcal{P}(\bar{v})$ and $\tilde{\mathcal{P}}(\bar{v})$, the PDFs of the Lévy walk system for $0<\alpha<1$, are presented in their general form. The two PDFs also show some similarities. As $\alpha \rightarrow 1$, both equate and give a Dirac delta function (see appendix of [25]),

$$
\lim _{\alpha \rightarrow 1} \mathcal{P}(\bar{v})=\lim _{\alpha \rightarrow 1} \tilde{\mathcal{P}}(\bar{v})=-\frac{1}{\pi} \lim _{\epsilon \rightarrow 0} \operatorname{Im}\left[\int_{-\infty}^{\infty} d v F(v)(\bar{v}-v+i \epsilon)\right]^{-1}=\delta(\bar{v}-\langle v\rangle) .
$$


In the opposite limit, $\alpha \rightarrow 0$, the particle is stuck in one state most of the time since the sojourn time has a very fat tail. For the jump model, the particle is stranded for almost the entire measurement time causing $x(t) / t \rightarrow 0$ in the long time limit. Indeed applying inverse Laplace and Fourier transforms on Eq. (8.7) and inserting to Eq. (8.8) we find

$$
\lim _{\alpha \rightarrow 0} \tilde{\mathcal{P}}(\bar{v})=\delta(\bar{v})
$$

In the velocity model, and in the $\alpha \rightarrow 0$ limit, the particle travels most the observation time with a constant velocity $v$ whose value is drawn from $F(v)$. By applying the same inverse transforms on Eq. (8.2) as above and inserting into Eq. (8.3) we find

$$
\lim _{\alpha \rightarrow 0} \mathcal{P}(\bar{v})=F(\bar{v}) .
$$

The two PDFs, Eqs. (8.6) and (8.9), have a striking difference at the origin, where the jump model PDF diverges. For a special case of a symmetric PDF $F(v)=F(-v)$, notice that $H_{\alpha}^{<}(0)=H_{\alpha}^{>}(0)=\left\langle|v|^{\alpha}\right\rangle / 2$ and $H_{\alpha-1}^{<}(0)=H_{\alpha-1}^{>}(0)=\left\langle|v|^{\alpha-1}\right\rangle / 2$, which we assume exists. The $\operatorname{PDF} \mathcal{P}(\bar{v}=0)$ is calculated in the leading order, using Eq. (8.6),

$$
\mathcal{P}(\bar{v}=0)=\frac{\tan \left(\frac{\pi \alpha}{2}\right)}{\pi} \frac{\left\langle|v|^{\alpha-1}\right\rangle}{\left\langle|v|^{\alpha}\right\rangle}
$$

where $0<\alpha<1$. For the jump model we use Eq. (8.9) to find

$$
\tilde{\mathcal{P}}(\bar{v}) \sim \frac{\tan \left(\frac{\pi \alpha}{2}\right)}{\pi} \frac{1}{\left\langle|v|^{\alpha}\right\rangle}|\bar{v}|^{\alpha-1} .
$$

We now turn to the statistical mean and the variance. They are calculated by applying the method of [40] to Eqs. (8.2) and (8.7). We define $\xi=-i k / u$ and

$$
\frac{\left\langle x^{q}\right\rangle}{t^{q}}=\left\{\begin{array}{cl}
\left.\frac{(-1)^{q}}{q !} \frac{d^{q}}{d \xi^{q}} \frac{\left\langle[1+\xi v]^{\alpha-1}\right\rangle}{\left\langle[1+\xi v]^{\alpha}\right\rangle}\right|_{\xi=0} & \text { velocity model } \\
\left.\frac{(-1)^{q}}{q !} \frac{d^{q}}{d \xi^{q}} \frac{1}{\left\langle[1+\xi v]^{\alpha}\right\rangle}\right|_{\xi=0} & \text { jump model. }
\end{array}\right.
$$

The first cumulant is calculated

$$
\langle x\rangle=\left\{\begin{aligned}
\langle v\rangle t & \text { velocity model } \\
\alpha\langle v\rangle t & \text { jump model. }
\end{aligned}\right.
$$

The second cumulant, i.e., the variance, is

$$
\left\langle x^{2}\right\rangle-\langle x\rangle^{2}=\left\{\begin{array}{rr}
(1-\alpha)\left[\left\langle v^{2}\right\rangle-\langle v\rangle^{2}\right] t^{2} & \text { velocity model, } \\
\alpha(1-\alpha) \frac{\left\langle v^{2}\right\rangle}{2} t^{2} & \text { jump model. }
\end{array}\right.
$$

The abrupt displacement events of the jump model affect the mean and the variance due to the particle's stickiness in contrast to the velocity model. This is especially noticeable near $\alpha=0$.

\subsection{Numerical examples}

Some example cases will now be discussed. The first case is the symmetric two state model given by the velocity PDF $F_{\mathrm{TS}}(v)$, Eq. (2.1). Using Eqs. (8.4) and (8.5) in (8.6) we find Lamperti's PDF [40]

$$
\mathcal{P}_{\mathrm{TS}}(\bar{v})= \begin{cases}\frac{\sin (\pi \alpha)}{\pi} \frac{\frac{2}{v_{\mathrm{C}}}\left[1-\left(\frac{\bar{v}}{v_{\mathrm{c}}}\right)^{2}\right]^{\alpha-1}}{\left[1+\frac{\bar{v}}{v_{\mathrm{c}}}\right]^{2 \alpha}+\left[1-\frac{\bar{v}}{v_{\mathrm{C}}}\right]^{2 \alpha}+2 \cos (\pi \alpha)\left[1-\left(\frac{\bar{v}}{v_{\mathrm{C}}}\right)^{2}\right]^{\alpha}} & \text { for }|\bar{v}| \leq v_{\mathrm{c}} \\ 0 & \text { for }|\bar{v}|>v_{\mathrm{c}}\end{cases}
$$




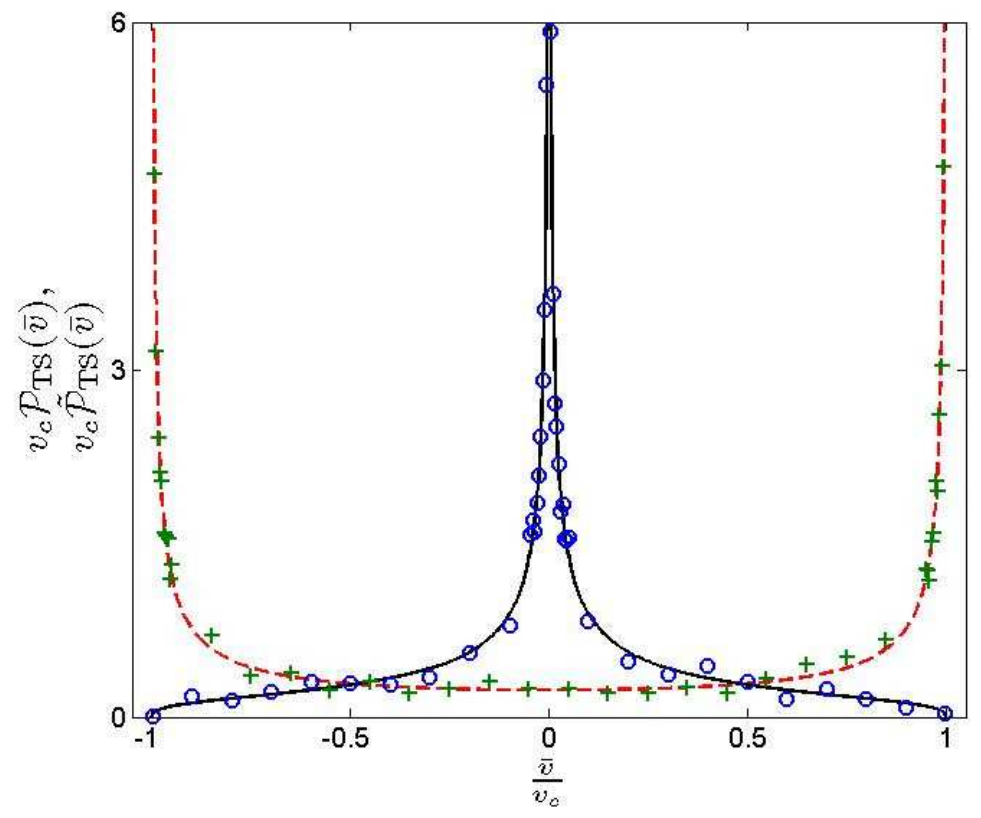

FiguRE 7. (color online) Simulations (symbols) versus theory (lines) for the two state model with $\alpha=0.4$. The velocity attains values \pm 1 with equal probability. Simulation results are given for the velocity $(+)$ and jump (o) models are compared with the theoretical results $(8.18,8.19)$ respectively. Numerically obtained PDFs found by sampling over $10^{5}$ realizations.

and by Eq. (8.9)

$$
\tilde{\mathcal{P}}_{\mathrm{TS}}(\bar{v})= \begin{cases}\frac{\sin (\pi \alpha)}{\pi} \frac{\frac{2}{v_{\mathrm{c}}}\left|\frac{\bar{v}}{v_{\mathrm{c}}}\right|^{\alpha-1}\left[1-\mid \frac{\bar{v}}{v_{\mathrm{C}}}\right]^{\alpha}}{\left[1+\frac{\bar{v}}{v_{\mathrm{C}}}\right]^{2 \alpha}+\left[1-\frac{\bar{v}}{v_{\mathrm{c}}}\right]^{2 \alpha}+2 \cos (\pi \alpha)\left[1-\left(\frac{\bar{v}}{v_{\mathrm{c}}}\right)^{2}\right]^{\alpha}} & \text { for }|\bar{v}| \leq v_{\mathrm{c}}, \\ 0 & \text { for }|\bar{v}|>v_{\mathrm{c}} .\end{cases}
$$

The second case is a system with a biased two state PDF

$$
F(v)=\frac{1}{1+R} \delta\left(v+v_{\mathrm{c}}\right)+\frac{R}{1+R} \delta\left(v-v_{\mathrm{c}}\right),
$$

where $R>0$ is the asymmetry parameter. Specifically, in the previous example we set $R=1$. Straightforward calculations yield

$$
\mathcal{P}_{\text {bias TS }}(\bar{v})=\frac{\sin (\pi \alpha)}{\pi} \frac{\frac{2 R}{v_{\mathrm{c}}}\left[1-\left(\frac{\bar{v}}{v_{\mathrm{c}}}\right)^{2}\right]^{\alpha-1}}{\left[1+\frac{\bar{v}}{v_{\mathrm{c}}}\right]^{2 \alpha}+R^{2}\left[1-\frac{\bar{v}}{v_{\mathrm{c}}}\right]^{2 \alpha}+2 R \cos (\pi \alpha)\left[1-\left(\frac{\bar{v}}{v_{\mathrm{c}}}\right)^{2}\right]^{\alpha}},
$$

and

$$
\tilde{\mathcal{P}}_{\text {biasTS }}(\bar{v})=\frac{\sin (\pi \alpha)}{\pi} \frac{\frac{1+R}{v_{\mathrm{c}}}\left|\frac{\bar{v}}{v_{\mathrm{c}}}\right|^{\alpha-1}\left[1-\left|\frac{\bar{v}}{v_{\mathrm{c}}}\right|\right]^{\alpha}[1+(R-1) I(\bar{v}>0)]}{\left[1+\frac{\bar{v}}{v_{\mathrm{c}}}\right]^{2 \alpha}+R^{2}\left[1-\frac{\bar{v}}{v_{\mathrm{c}}}\right]^{2 \alpha}+2 R \cos (\pi \alpha)\left[1-\left(\frac{\bar{v}}{v_{\mathrm{c}}}\right)^{2}\right]^{\alpha}} .
$$




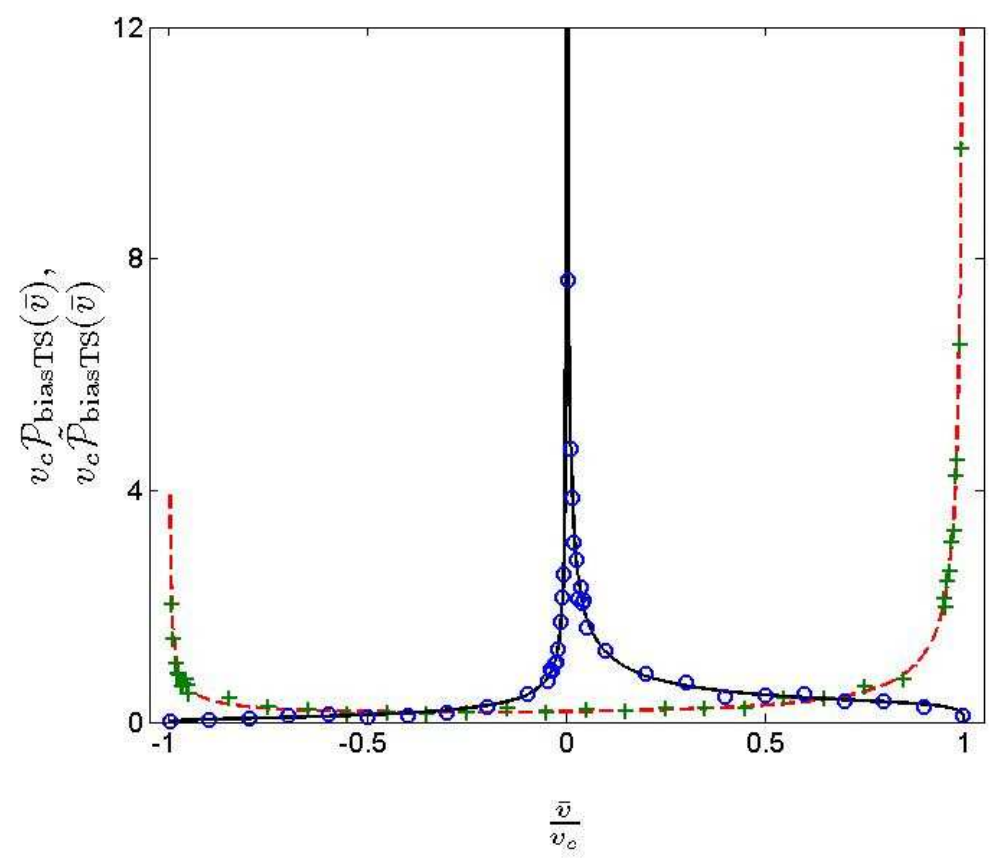

Figure 8. (color online) Simulations (symbols) and theory (lines) for the two state system with $\alpha=0.4$ and $v_{\mathrm{c}}=1$. The velocity attains values $v=1$ and $v=-1$ with probability 0.7 and 0.3 , respectively [i.e. $R=7 / 3$ in Eq. (8.20)]. Simulation results are presented for the velocity $(+)$ and jump (o) models. The lines are the theoretical PDFs, Eqs. (8.21) and (8.22), respectively. The velocity model exhibits ballistic peaks diverging on $\bar{v}= \pm v_{\mathrm{c}}$ while for the jump model, divergence is found at the origin $\bar{v}=0$. Numerically obtained PDFs found by sampling over $10^{5}$ realizations.

Eqs. (8.18) and (8.21) are the Lamperti distribution [9, 25, 40].

The third case is a system with an exponential PDF

$$
F_{\exp }(v)=\exp \left(-\sqrt{2}|\bar{v}| / v_{\mathrm{c}}\right) /\left[\sqrt{2} v_{\mathrm{c}}\right]
$$

where $v_{\mathrm{c}}>0$. A straight forward calculation of Eqs. (8.4) and (8.5) for $\bar{v}>0$ yields

$$
H_{\alpha, \exp }^{<}(\bar{v})=\frac{\left(v_{\mathrm{c}}\right)^{\alpha} e^{-\frac{\sqrt{2} \bar{v}}{v_{\mathrm{c}}}}}{2^{1+\frac{\alpha}{2}}} \Gamma(1+\alpha)
$$

and

$$
H_{\alpha, \exp }^{>}(\bar{v})=\frac{\left(v_{\mathrm{c}}\right)^{\alpha} e^{-\frac{i \pi \alpha}{2}}}{2^{1+\frac{\alpha}{2}}}\left[\Gamma\left(1+\alpha, \frac{\sqrt{2} \bar{v}}{v_{\mathrm{c}}}\right) e^{\frac{\sqrt{2} \bar{v}}{v_{\mathrm{c}}}+\frac{i \pi \alpha}{2}}-\gamma\left(1+\alpha,-\frac{\sqrt{2} \bar{v}}{v_{\mathrm{c}}}\right) e^{-\frac{\sqrt{2} \bar{v}}{v_{\mathrm{c}}}-\frac{i \pi \alpha}{2}}\right],
$$

where $\Gamma(\alpha, x)=\int_{x}^{\infty} d y e^{-y} y^{\alpha-1}$ and $\gamma(\alpha, x)=\Gamma(\alpha)-\Gamma(\alpha, x)$ are the incomplete gamma functions [4]. Using the symmetric properties of the exponential PDF we can calculate $\mathcal{P}^{\exp }(\bar{v})$ and $\tilde{\mathcal{P}}^{\exp }(\bar{v})$ on the RHS using Eqs. (8.6) and (8.9). The asymptotic expressions of the PDFs is obtained via approximations. 


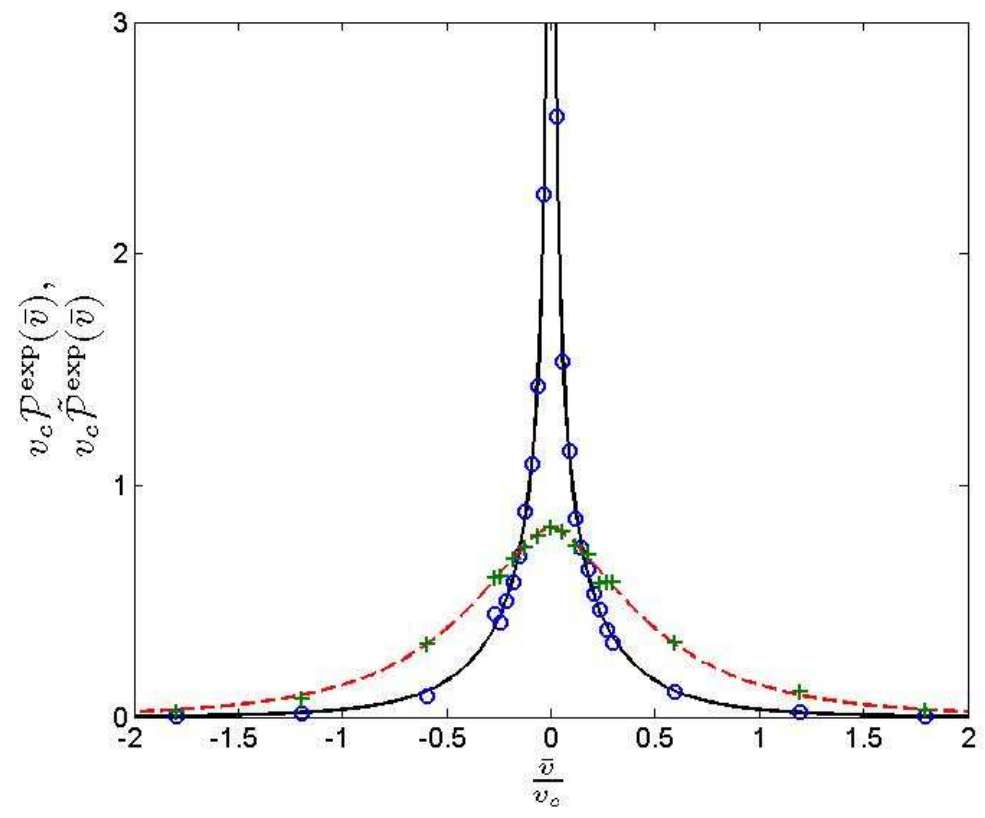

Figure 9. (color online) Simulations (symbols) and theory (lines) for the model with the symmetrical exponential velocity PDF, Eq. (8.23) with $\alpha=0.4$. Simulation results are presented for the velocity $(+)$ and jump (o) models. The lines are the theoretical PDFs. Numerically obtained PDFs found by sampling over $10^{5}$ realizations.

Using integration by parts one proves that leading terms are $\left.\Gamma(\alpha, x)\right|_{x \gg 1} \sim x^{\alpha-1} e^{-x}+O\left(x^{\alpha-2}\right)$ and $\left.\gamma(\alpha,-x)\right|_{x \gg 1} \sim e^{i \pi \alpha} x^{\alpha-1} e^{x}$. The auxiliary function, Eq. (8.25), is expressed by

$$
\left.H_{\alpha, \exp }^{>}(|\bar{v}|)\right|_{|\bar{v}| \gg 1}=|\bar{v}|^{\alpha}
$$

for $\bar{v}>0$. Thus

$$
\left.\mathcal{P}^{\exp }(|\bar{v}|)\right|_{|\bar{v}| \gg v_{0}} \sim \frac{\sin (\pi \alpha)}{\pi} \frac{\Gamma(\alpha)\left(v_{\mathrm{c}}\right)^{\alpha-1}}{2^{\frac{\alpha+1}{2}}} \frac{e^{-\frac{\sqrt{2}|\bar{v}|}{v_{\mathrm{c}}}}}{|\bar{v}|^{\alpha}}
$$

and

$$
\widetilde{\mathcal{P}}^{\exp }(|\bar{v}|)||_{|\bar{v}| \gg v_{0}} \sim \frac{\sin (\pi \alpha)}{\pi} \frac{\Gamma(\alpha+1)\left(v_{\mathrm{c}}\right)^{\alpha}}{2^{1+\frac{\alpha}{2}}} \frac{e^{-\frac{\sqrt{2}|\bar{v}|}{v_{\mathrm{c}}}}}{|\bar{v}|^{\alpha+1}} .
$$

The fourth case is a system with a constant velocity $v_{\mathrm{c}}$. Its $\mathrm{PDF}$ is $F(v)=\delta\left(v-v_{\mathrm{c}}\right), v_{\mathrm{c}}>0$. In the velocity model the particle moves constantly with that velocity, turning the time averaged velocity to a constant independent of $\alpha$. The PDF, Eq. (8.3) is simplified using Eq. (8.10)

$$
\mathcal{P}_{v_{\mathrm{c}}}(\bar{v})=-\frac{1}{\pi} \lim _{\epsilon \rightarrow 0} \operatorname{Im}\left(\bar{v}-v_{\mathrm{c}}+i \epsilon\right)^{-1}=\delta\left(\bar{v}-v_{\mathrm{c}}\right) .
$$

In the jump model the particle jumps only at the end of each event, causing the position is proportional to the sum of the waiting times, $\widetilde{x}(t)=v_{\mathrm{c}} t_{n}$ for $n$ events. The PDF, Eq. (8.8) for positive $\bar{v}$ is

$$
\tilde{\mathcal{P}}_{v_{\mathrm{c}}}(\bar{v})=-\frac{|\bar{v}|^{\alpha-1}}{\pi} \lim _{\epsilon \rightarrow 0} \operatorname{Im}\left(\bar{v}-v_{\mathrm{c}}+i \epsilon\right)^{-\alpha},
$$


where the zero order in $\epsilon$ is

$$
\begin{gathered}
\operatorname{Im}\left(\bar{v}-v_{\mathrm{c}}+i \epsilon\right)^{-\alpha} \sim\left|\bar{v}-v_{\mathrm{c}}\right|^{\alpha} \operatorname{Im}\left\{\begin{array}{r}
e^{-i \pi \alpha} \text { for } \bar{v}<v_{\mathrm{c}}, \\
1 \text { for } \bar{v}>v_{\mathrm{c}},
\end{array}\right. \\
=\left\{\begin{array}{r}
-\left|\bar{v}-v_{\mathrm{c}}\right|^{\alpha} \sin (\pi \alpha) \text { for } \bar{v}<v_{\mathrm{c}}, \\
0 \text { for } \bar{v}>v_{\mathrm{c}} .
\end{array}\right.
\end{gathered}
$$

yielding

$$
\tilde{\mathcal{P}}_{v_{\mathrm{c}}}(\bar{v})=\frac{\sin (\pi \alpha)}{\pi}|\bar{v}|^{\alpha-1}\left|v_{\mathrm{c}}-\bar{v}\right|^{-\alpha},
$$

for $0<\bar{v}<v_{\text {c }}$ otherwise $\tilde{\mathcal{P}}(\bar{v})=0$. This Beta distribution function corresponds to the statistics of the backward recurrence time in [25] since $\bar{v} \propto t_{n} / t$.

\section{Summary}

We have analyzed two complementary densities of the Lévy walk. When the average flight time is finite $\alpha>1$ we find dual scaling. In the super diffusive phase, $1<\alpha<2$, the bulk fluctuations are described by Lévy's central limit theorem. Naively, this implies the blow up of the mean square displacement $\left\langle x^{2}\right\rangle=\infty$, since the variance of the Lévy distribution is infinite. This is clearly unphysical for any model with a typical finite velocity. The infinite density is complementary to the Lévy central limit theorem and it exhibits ballistic scaling. This non-normalised density correctly describes moments, or more generally observables, which are integrable. For example $|x|^{q}$ with $q>\alpha$ is integrable (non-integrable) with respect to the infinite (Lévy) density respectively. Hence the moments $\left\langle|x|^{q}\right\rangle$ with $q>\alpha$ (or $\left.0<q<\alpha\right)$ are obtained from the infinite (Lévy) density, respectively. In that sense the infinite and Lévy densities are complementary. Both of them together yield the description of the packet of particles, and the phenomenon of strong anomalous diffusion.

The Mellin transform technique, developed in this article, yields an explicit equation for the infinite density. In many applications the analysis of rare fluctuations is made with the theory of large deviations, however that theory does not apply in our case $[49,50]$. The technique developed here, was used to analyse two models. In the velocity model the particle moves with a constant velocity between collision events, while in the jump model the particle is static most of the time, performing sudden jumps at renewal events. Both models yield an infinite density indicating that the Mellin transform approach is general.

We have found three behaviors corresponding to the three phases of the Lévy walk model:

(i) For $0<\alpha<1$ we have mono-scaling and the density is described by tools borrowed from the theory of weak ergodicity breaking. The limit theorems for the density are related to the arcsine law and Lamperti's distribution.

(ii) For $1<\alpha<2$ the Lévy density describes the central part of the packet of particle and it matches the infinite density describing the tail of the density. Here we get a uniform approximation describing both the rare and typical fluctuations.

(iii) When $2<\alpha$ the diffusion is normal in the mean square displacement sense. The central part of the density is described by the Gaussian central limit theorem, while the outer part by the infinite density. The two solutions do not match in the central region. Hence as suggested in the text, further work is needed to obtain a uniform approximation.

The perspective ways for further research are many even with a stochastic framework. For example, we have considered a process that started at time $t=0$. If the process has been initiated at time $t=-\infty$, the system reaches a steady state at time $t=0$ when the observation begins. Such an equilibrium state is found only when $\alpha>1$. What is the infinite density in that case? (if it exists). Another interesting case is to investigate a model where the increments of displacement grows like time to the power of $\beta \neq 1$. A partial answer to this case can be found in Ref. [19]. 
Acknowledgements. This work was supported by the Israel Science Foundation (AR and EB) and the German Excellence Initiative Nanosystems Initiative Munich"(SD and PH). EB thanks the Alexander von Humboldt foundation for its support.

\section{A. The Montroll-Weiss equation for the velocity model}

The Montroll-Weiss equation describes the CTRW in the $(k, u)$ space. We wish to calculate the PDF, $P(x, t)$. To do so, we work in the Fourier-Laplace space, where the expression is easier to determine. The PDF of $x(t)$ is composed of mutually exclusive cases of different number of events, $n,[25]$

$$
P(x, t)=\sum_{n=0}^{\infty}\left\langle I\left(t_{n}<t<t_{n+1}\right) \delta[x-x(t)]\right\rangle,
$$

where $I(\cdot)=1$ if the argument in the parenthesis is true or 0 if it is false, $\delta(\cdot)$ is the Dirac delta and $\langle\cdot\rangle$ represents statistical averaging over the sojourn times and the velocities. In what follows we use a convention of indicating the space we are working in by the function's variables in the parenthesis, where $(k, x)$ is the Fourier conjugate pair and $(u, t)$ is the Laplace conjugate pair.

As a first step, we determine the characteristic function, $P(k, u)$, using Eqs. (A.1) and (2.5),

$$
P(k, u)=\left\langle e^{i k x-u t}\right\rangle=\int_{0}^{\infty} \mathrm{d} t e^{-u t} \int_{-\infty}^{\infty} \mathrm{d} x e^{i k x} P(x, t)=\sum_{n=0}^{\infty}\left\langle\int_{t_{n}}^{t_{n+1}} \mathrm{~d} t e^{i k x(t)-u t}\right\rangle .
$$

Inserting Eq. (2.4) we perform the integral on the RHS of Eq. (A.2) using $t_{n+1}-t_{n}=\tau_{n+1}$

$$
\begin{gathered}
P(k, u)=\sum_{n=0}^{\infty}\left\langle\frac{1-e^{-\left(u-i k v_{n}\right) \tau_{n+1}}}{u-i k v_{n}} e^{-\sum_{j=1}^{n}\left(u-i k v_{j-1}\right) \tau_{j}}\right\rangle= \\
\left\langle\frac{1-e^{-\left(u-i k v_{0}\right) \tau_{1}}}{u-i k v_{0}}\right\rangle+\sum_{n=1}^{\infty}\left\langle\frac{1-e^{-\left(u-i k v_{n}\right) \tau_{n+1}}}{u-i k v_{n}} \prod_{j=1}^{n} e^{-\left(u-i k v_{j-1}\right) \tau_{j}}\right\rangle .
\end{gathered}
$$

The iid trait and the definition $\hat{\psi}(u)=\left\langle e^{-u \tau}\right\rangle$ enable transforming Eq. (A.3) to

$$
\left\langle\frac{1-e^{-(u-i k v) \tau}}{u-i k v}\right\rangle \sum_{n=0}^{\infty}\left\langle e^{-(u-i k v) \tau}\right\rangle^{n}=\left\langle\frac{1-\hat{\psi}(u-i k v)}{u-i k v}\right\rangle \sum_{n=0}^{\infty}\langle\hat{\psi}(u-i k v)\rangle^{n} .
$$

This geometric series sum is convergent and yields the known Montroll-Weiss equation [7,29,55] and $\langle\cdot\rangle$ indicates here averaging with respect to $\left\{v_{j}\right\}_{j=0,1, \ldots}$ only.

An alternate form is derived by recalling the Laplace transform identity found using integration by parts,

$$
\int_{0}^{\infty} \mathrm{d} \tau e^{-u \tau} \int_{\tau}^{\infty} \mathrm{d} y \psi(y)=\frac{1-\hat{\psi}(u)}{u}
$$

which expresses the probability to stay in a state until time $t$. Inserting this identity into Eq. (2.6) yields

$$
P(k, u)=\frac{\int_{0}^{\infty} d \tau \int_{-\infty}^{\infty} d v e^{-(u-i k v) \tau} F(v) \int_{\tau}^{\infty} d y \psi(y)}{1-\int_{0}^{\infty} d \tau \int_{-\infty}^{\infty} d v e^{-(u-i k v) \tau} F(v) \psi(\tau)}
$$

a more common form.

\section{B. The Montroll-Weiss equation for the jump model}

The jump model is simpler than the velocity model. The dynamics takes place at discrete times $\left\{t_{n}\right\}_{n=1,2, \ldots}$, i.e., the transition times between the renewal events (see Fig. 2). Following Sec. 2.1 we derive $\tilde{P}(x, t)$, the PDF of $x$ at time $t$,

$$
\tilde{P}(x, t)=\sum_{n=0}^{\infty}\left\langle I\left(t_{n}<t<t_{n+1}\right) \delta[x-\tilde{x}(t)]\right\rangle,
$$




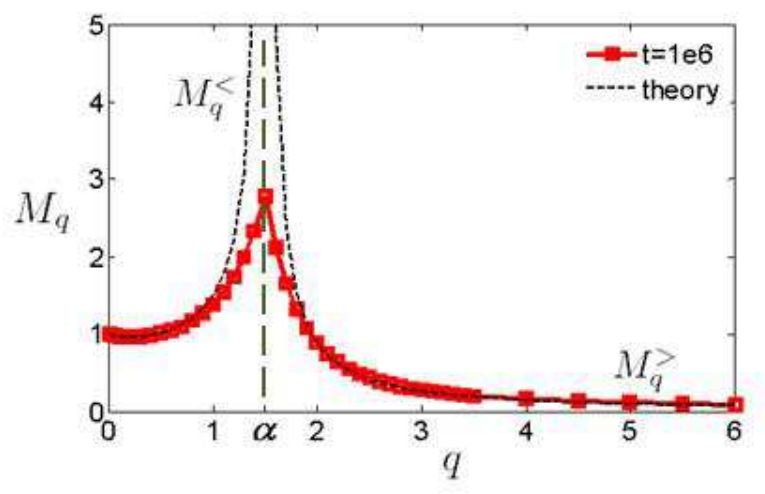

Figure 10. Asymptotic moment amplitudes, Eq. (C.4), for the two-state model (dashed lines) are compared with the results of the statistical sampling for $t=10^{6}$ (squares). Theory predicts a singularity at the point $q=\alpha$, corresponding to a first order pole. The parameters of the model are $\alpha=1.5, A=|\Gamma(1-\alpha)|, v_{\mathrm{c}}= \pm 1$. The number of realizations is $10^{6}$.

where $\tilde{x}(t)$ is taken from Eq. (2.7). The Fourier-Laplace transform of $\tilde{P}(x, t)$ is

$$
\begin{aligned}
\tilde{P}(k, u) & =\sum_{n=0}^{\infty}\left\langle\frac{1-e^{-u \tau_{n+1}}}{u} e^{-\sum_{j=1}^{n}\left(u-i k v_{j-1}\right) \tau_{j}}\right\rangle \\
& =\frac{1-\hat{\psi}(u)}{u} \frac{1}{1-\langle\hat{\psi}(u-i k v)\rangle},
\end{aligned}
$$

as in Eq. (2.8).

\section{The complementing densities}

Up to now we were focusing on the long time limit, and thus concentrating on the characteristic function $P(k, u)$, Eq. (3.1). This is not enough to attain a comprehensive analytical expression for the full PDF in the $(x, t)$ phase space. Later, we delved into long time limit solutions and partitioned the PDF to a central region function, $P_{\text {cen }}(x, t)$, a Lévy stable distribution, Eq. (5.2), and an outer region function, $t^{-\alpha} \mathcal{I}_{\mathrm{d}}(x / t)$, which approximates $P(x, t)$ in the exterior region $|x| \sim t$. The results indicate that in the large $t$ limit $P_{\text {cen }}(x, t)$ depends on $\left\langle\left|v^{\alpha}\right|\right\rangle$ and the asymptotic form of $\psi(\tau)$, while the external region depends on the latter and the entire velocity PDF, $F(v)$. Both densities are independent of initial conditions in the long time limit as expected. Between the central and the outer distributions there is a power law dependence, Eq. (5.3), which is the tail of the Lévy function and the near-origin divergence of the infinite density. This matching indicates that the two distributions complement each other in extracting the statistical properties of the PDF, $P(x, t)$. We will show how the two distributions are mutually exclusive in the following cases of moment calculations.

The behavior of the $P(x, t)$ in the long time limit can be divided to three from the center, $x \sim 0$, outward: (i) $P_{\text {cen }}(x, t)$, (ii) the matched intermediate power law region and (iii) $t^{-\alpha} \mathcal{I}_{\mathrm{d}}(x / t)$. We can establish two crossover velocities which delimit the matched power law region, $v_{c 1} t<|x|<v_{c 2} t$. We will calculate half the moments value for the sake of simplicity (owing to the symmetry of the system)

$$
\int_{0}^{\infty} x^{q} P(x, t) d x=\underbrace{\int_{0}^{v_{c 1} t} x^{q} P_{\text {cen }}(x, t) d x}_{\text {center region }}+\underbrace{K_{\alpha} c_{\alpha} t \int_{v_{c 1} t}^{v_{c 2} t} \frac{x^{q} d x}{x^{1+\alpha}}}_{\text {intermediate region }}+\underbrace{t^{-\alpha} \int_{v_{c 2} t}^{\infty} x^{q} \mathcal{I}_{\mathrm{d}}(x / t) d x}_{\text {ballistic outer region }}
$$


The crossover velocities $v_{c 1}$ and $v_{c 2}$ can be estimated. The key issue is their time dependence which indicates which region dominantly dictates the moment calculation. The first, $v_{c 1}$, is the shift from inner Lévy distribution to asymptotic power law, was approximated in [41] as the intersection between $P_{\text {center }}(x=0, t)=L_{\alpha}(0) /\left[K_{\alpha} t\right]^{1 / \alpha}=\Gamma\left(1+\alpha^{-1}\right) /\left[K_{\alpha} t\right]^{1 / \alpha}$ and the intermediate power law region $t^{-\alpha} K_{\alpha} c_{\alpha} v_{c 1}^{-1-\alpha}$, meaning $v_{c 1} t \sim\left(K_{\alpha} t\right)^{1 / \alpha}$. The second crossover velocity, $v_{c 2}$, is the end of the nearorigin divergence of the ID. It is derived by equating the first two terms in the expansion of the series, Eq. (4.12), which yields $v_{c 2} \sim\left\langle|v|^{\alpha}\right\rangle /\left\langle|v|^{\alpha-1}\right\rangle$. Inserting our estimations yields

$$
\begin{aligned}
\int_{0}^{\infty} x^{q} P(x, t) d x & =\int_{0}^{\left(K_{\alpha} t\right)^{1 / \alpha}} x^{q} P_{\operatorname{cen}}(x, t) d x+K_{\alpha} c_{\alpha} t \int_{\left(K_{\alpha} t\right)^{1 / \alpha}}^{\frac{\left\langle|v|^{\alpha}\right\rangle}{\langle\langle v-1\rangle} t} \frac{x^{q} d x}{x^{1+\alpha}}+t^{-\alpha} \int_{\frac{\left\langle\left. v\right|^{\alpha}\right\rangle}{\left\langle|v|^{\alpha-1\rangle}\right.} t}^{\infty} x^{q} \mathcal{I}_{\mathrm{d}}(x / t) d x \\
& =\left(K_{\alpha} t\right)^{q / \alpha} \int_{0}^{1} \zeta^{q} L_{\alpha}(\zeta) d \zeta+K_{\alpha} c_{\alpha} t \int_{\left(K_{\alpha} t\right)^{1 / \alpha}}^{\frac{\left\langle\left\langle v \alpha^{\alpha}\right\rangle\right.}{\left\langle|v|^{\alpha-1} t\right.} t} \frac{x^{q} d x}{x^{1+\alpha}}+t^{q+1-\alpha} \int_{\frac{\left\langle|v|^{\alpha}\right\rangle}{\left\langle|v|^{\alpha-1}\right\rangle}}^{\infty} \bar{v}^{q} \mathcal{I}_{\mathrm{d}}(\bar{v}) d \bar{v}
\end{aligned}
$$

where $\zeta=\left(K_{\alpha} t\right)^{-1 / \alpha} x$. The intermediate power law region can be written in two ways:

$$
K_{\alpha} c_{\alpha} t \int_{\left(K_{\alpha} t\right)^{1 / \alpha}}^{\frac{\left\langle|v|^{\alpha}\right\rangle}{\left\langle|v|^{\alpha-1}\right\rangle} t} \frac{x^{q} d x}{x^{1+\alpha}}=c_{\alpha}\left(K_{\alpha} t\right)^{q / \alpha} \int_{1}^{\frac{\left\langle\left\langle\left. v\right|^{\alpha}\right\rangle\right.}{K_{\alpha}^{1 / \alpha}\left\langle|v|^{\alpha-1}\right\rangle}} t^{1-1 / \alpha} \frac{\zeta^{q} d \zeta}{\zeta^{1+\alpha}}=K_{\alpha} c_{\alpha} t^{q+1-\alpha} \int_{K_{\alpha}^{1 / \alpha} t^{1 / \alpha-1}}^{\frac{\left\langle\left\langle\left. v\right|^{\alpha}\right\rangle\right.}{\left\langle|v|^{\alpha-1}\right\rangle}} \frac{\bar{v}^{q} d \bar{v}}{\bar{v}^{1+\alpha}} .
$$

In the long time, the moment's order $q$ determines both the time dependence of the moment and the integral's limits. At $t \rightarrow \infty$ the integral's limits become $(1, \infty)$ for $q<\alpha$ with a time dependence of $t^{q / \alpha}$, meaning the Lévy phase determines the moment's calculation. For $q>\alpha$ the limits are $\left(0,\left\langle|v|^{\alpha}\right\rangle /\left\langle|v|^{\alpha-1}\right\rangle\right)$ with a $t^{q+1-\alpha}$ dependence. Thus, in the latter case the infinite density determines alone the moment's calculation. At $q=\alpha$, we have a critical value, where the intermediate region integral converges to one form. This integrand is the matching curve, Eq. (5.3), of both the central limit theorem and the ID. It contributes to either distributions as a fat tail or a power law divergence at the origin (see Fig. 4). The moment amplitudes are defined using Eqs. (4.4) and a calculation using the Lévy PDF, Eq. (5.2),

$$
\begin{aligned}
& M_{\alpha}^{<}=\frac{\left\langle\left|x^{q}(t)\right|\right\rangle}{t^{q / \alpha}} \sim \frac{\left(K_{\alpha}\right)^{\frac{q}{\alpha}} \Gamma(1-q / \alpha)}{\Gamma(1-q) \cos (\pi q / 2)} \text { for } 0 \leq q<\alpha \\
& M_{\alpha}^{>}=\frac{\left\langle|x(t)|^{q}\right\rangle}{t^{q+1-\alpha}} \sim \frac{2 K_{\alpha} c_{\alpha}}{\alpha\left\langle|v|^{\alpha}\right\rangle} \frac{q\left\langle|v|^{m}\right\rangle}{(q-\alpha)(q-\alpha+1)} \text { for } \alpha<q
\end{aligned}
$$

Notice that both amplitudes have a 1st order pole at $q=\alpha$ (see Fig. 10). Only the moments of order $q>\alpha$ reflect uniquely the velocity $\mathrm{PDF}, F(v)$. For $q<\alpha$, the central limit theorem obscures $F(v)$ as it only contains partial information, $K_{\alpha} \propto\left\langle|v|^{\alpha}\right\rangle$. Now we can assign values characterizing strong anomalous diffusion to Eq. (1.3) with $d_{1}=1 / \alpha, d_{2}=1, d_{3}=1-\alpha$ and $q_{c}=\alpha$ [See Fig. 1].

\section{References}

[1] T. Akimoto, Distributional responses to biases in deterministic super-diffusion. Phys. Rev. Lett. 108 (2012) 164101.

[2] T. Akimoto, T. Miyagichi. Phase diagram in stored-energy-driven Lévy flight. J. Stat. Phys. 157 (2014) 515-530.

[3] K. H. Andersen, P. Castiglione, A. Mazzino, A. Vulpiani. Simple stochastic models showing strong anomalous diffusion. Eur. Phys. J. B. 18 (2000) 447-452.

[4] G. B. Arfken, H. J. Weber. Mathematical methods for physicists, Academic Press (1995).

[5] B. Baeumer, M.M. Meerschaert, J. Mortensen. Space time fractional derivative operators. Proc. Amer. Math. Soc. (ISSN: 0002-9939) 133 (8) (2005) 2273-2282.

[6] E. Barkai, E. Aghion, D. A. Kessler. From the Area under the Bessel Excursion to Anomalous Diffusion of Cold Atoms. Phys. Rev. X., 4 (2014) 021036.

[7] E. Barkai, J. Klafter. Anomalous diffusion in the strong scattering limit: a Lévy walk approach Proceedings of a workshop held in Carry-Le-Rouet, (1997) S. Benkadda and G. M. Zaslavsky Editors Springer (Berlin).

[8] P. Becker-Kern, M. Meerschaert, H. Scheffler. Limit theorems for coupled continuous time random walks. Ann. Probab., 32 (1) (2004) 730-756.

[9] G. Bel, E. Barkai. Weak ergodicity breaking in the continuous time random walk. Phys. Rev. Lett., 94 (2005) 240602.

[10] J. P. Bouchaud and A. Georges. Anomalous diffusion in disordered media: Statistical mechanism, models and physical applications. Phys. Rep., 195 (1998) 127-293. 
[11] R. Burioni, L. Caniparoli, A. Vezzani. Lévy walks and scaling in quenched disordered media. Phys. Rev. E., 81 (2010) 060101.

[12] R. Burioni, G. Gradenigo, A. Sarracino, A. Vezzani, A. Vulpiani. Rare events and scaling properties in field-induced anomalous dynamics. J. Stat. Mech. Theory and Experiment, 09 (2013) P09022.

[13] G. Campagnola, K. Nepal, B. W. Schroder, O. B. Peersen, D. Krapf. Super diffusive motion of membrane targeting C2 domains. Scientific Reports, 5 (2015) 17721.

[14] B. A. Carreras, V. E. Lynch, D. E. Newman, G. M. Zaslavsky. Anomalous diffusion in a running sandpile model. Phys. Rev. E., 60, (1999) 4770.

[15] P. Castiglione, A. Mazzino, P. Muratore-Ginanneschi, A. Vulpiani. On strong anomalous diffusion. Physica D, 134 (1999) 75-93.

[16] Clearly, the strip of definition can be extended in some cases. For example, if $B(v)$ is a Gaussian then $M(1 / 2)$ is finite. The choice $1 \leq \operatorname{Re}(q)$ stems from normalization.

[17] L. Comtet. Advanced Combinatorics, D. Reidel Publishing Company (1974).

[18] P. de Anna, T. Le Borgne, M. Dentz, A. M. Tartakovsky, D. Bolster, P. Davy. Flow intermittency, Dispersion and correlated CTRW in Porous media. Phys. Rev. Lett., 110 (2013) 184502.

[19] M. Dentz, T. Le Borgne, D. R. Lester, F. P. J. de Barros. Scaling forms of particles densities for Lévy walks and strong anomalous diffusion. Phys. Rev. E., 92 (2015) 032128.

[20] A. Dhar, K. Saito, B. Derrida. Exact solution of a Levy walk model for anomalous heat transport. Phys. Rev. E., 87 (2013) 010103(R)

[21] S. Fedotov. Single integrodifferential wave equation for a Lévy walk. Phys. Rev. E., 93 (2016) 020101(R).

[22] W. Feller. An introduction to probability theory and its applications 2, John Wiley and Sons, New York (1971).

[23] D. Froemberg, M. Schmiedeberg, E. Barkai, V. Zaburdaev. Asymptotic densities of ballistic Lévy walks. Phys. Rev. E., 91 (2015) 022131.

[24] N. Gal, D. Weihs. Experimental evidence of strong anomalous diffusion in living cells. Phys. Rev. E., 81 (2010) 020903(R).

[25] C. Godréche, J. M. Luck. Statistics of the occupation time of renewal processes. J. Stat. Phys., 104 (2001) $489-524$.

[26] N. Hazut, S. Medalion, D. A. Kessler, E. Barkai. Fractional Edgeworth Expansion: Corrections to the Gaussian-Lévy Central Limit Theorem. Phys. Rev. E., 91 (2015) 052124.

[27] A. Jurlewicz, P. Kern, M. Meerschaert, H.-P. Scheffler. Fractional governing equations for coupled random walks. Comput. Math. Appl., (ISSN: 08981221) 64 (10) (2012) 3021-3036.

[28] D.A. Kessler, E. Barkai. Infinite covariant density for diffusion in logarithmic potential and optical lattices. Phys. Rev. Lett., 105 (2010) 120602.

[29] J. Klafter, A. Blumen, M. F. Shlesinger. Stochastic pathways to anomalous diffusion. Phys. Rev. A., 35 (1987) $3081-$ 3085.

[30] N. Korabel, E. Barkai. Pesin-Type Identity for Intermittent Dynamics with a Zero Lyapunov Exponent. Phys. Rev. Lett., 102 (2000) 050601.

[31] D. Krapf, G. Campagnola, K. Nepal, O. B. Peersen. Strange kinetics of bulk mediated diffusion in living cells. arXiv:1601.04198 [cond-mat.stat-mech] (2016).

[32] P. Lévy. Théorie de l'addition des variables aléatoires. (1937) Gauthiers-Villars, Paris.

[33] E. Lukacs. Applications of the Faá di Bruno's formula in mathematical statistics. American Mathematical Monthly, 62 (1955) 340-348.

[34] M Magdziarz, HP Scheffler, P Straka, P Zebrowski. Limit theorems and governing equations for Lévy walks Stochastic Processes and their Applications, 125 (2015) 4021-4038.

[35] B. B. Mandelbrot, J. W. van Ness, Fractional Brownian Motions, Fractional Noises and Applications. SIAM Rev., 10 (1968) 422-437.

[36] G. Margolin, V. Protasenko, M. Kuno, E. Barkai. Photon Counting Statistics For Blinking CdSe-ZnS Quantum Dots: A Lévy Walk Process J. of Physical Chemistry B, 110 (2006) 19053-19060.

[37] R. Metzler, J. Klafter. Random walk's guide to anomalous diffusion: a fractional dynamics approach. Physics Reports, 339 (2000) 1-77.

[38] Ed. A. D. Poularikas. The Transform and Applications Handbook, CRC Press inc (1995).

[39] A. Rebenshtok, E. Barkai. Distribution of Time-Averaged Observables for Weak Ergodicity Breaking. Phys. Rev. Lett., 99 (2007) 210601.

[40] A. Rebenshtok, E. Barkai. Weakly non-ergodic Statistical Physics. J. Stat. Phys., 133, 565-586 (2008).

[41] A. Rebenshtok, S. Denisov, P. Hänggi, E. Barkai. Infinite densities in strong anomalous diffusion: beyond the central limit theorem. Phys. Rev. Lett., 112, (2014) 110601.

[42] A. Rebenshtok, S. Denisov, P. Hänggi, E. Barkai. Infinite densities for Lévy walks. Phys. Rev. E. 90 (2014), 062135.

[43] A. Rebenshtok, S. Denisov, P. Hänggi, E. Barkai. Reply to the comment on "Non-Normalizable Densities in Strong Anomalous Diffusion: Beyond the Central Limit Theorem". arXiv:1502.01749 [cond-mat.stat-mech] (2015).

[44] D. P. Sanders, H. Larralde. Occurrence of normal and anomalous diffusion in polygonal billiard channels. Phys. Rev. E., 73 (2006) 026205.

[45] J. H. P. Schulz, E. Barkai. Fluctuations around equilibrium laws in ergodic continuous-time random walks. Phys. Rev. E., 91, (2015) 062129.

[46] M. F. Shlesinger, B. J. West, J. Klafter. Levy dynamics of enhanced diffusion Application to turbulence. Phys. Rev. Lett., 58, (1987) 1100-1103. 
[47] F. D. Stefani, J. P. Hoogenboom, and E. Barkai, Beyond Quantum Jumps: Blinking Nano-scale Light Emitters. Physics Today, 62 (2009) 34-39.

[48] P. Straka, B. Henry. Lagging and leading coupled continuous time random walks renewal times and their joint limits. Stochast. Process. Appl. (ISSN: 03044149), 121 (2) (2011) 324-336.

[49] H. Touchette. The large deviation approach to statistical mechanics. Physics Reports, 478 (2009) 1-69.

[50] A. Vulpiani, F. Cecconi, M. Cencini, A. Puglisi, D. Vergni (Eds). Large deviations in physics: the legacy of the law of large numbers. Lecture notes in physics (2014).

[51] G. H. Weiss. Aspects and Applications of the Random Walk, North-Holland, Amsterdam (1994) .

[52] V. Zaburdaev, S. Denisov, P. Hänggi. Space-time velocity correlation function for random walks. Phys. Rev. Lett., 110, 170604 (2013).

[53] V. Zaburdaev, S. Denisov, P. Hänggi. Perturbation spreading in many-particle systems: a random walk approach. Phys. Rev. Lett., 106 (2011) 180601. ibid, Phys. Rev. Lett., 109, 069903 (2012).

[54] V. Zaburdaev, S. Denisov, J. Klafter. Lévy walks. Rev. Mod. Phys., 87 (2015) 483.

[55] G. Zumofen, J. Klafter. Scale-invariant motion in intermittent chaotic systems. Phys. Rev. E., 47 (1993) $851-863$. 